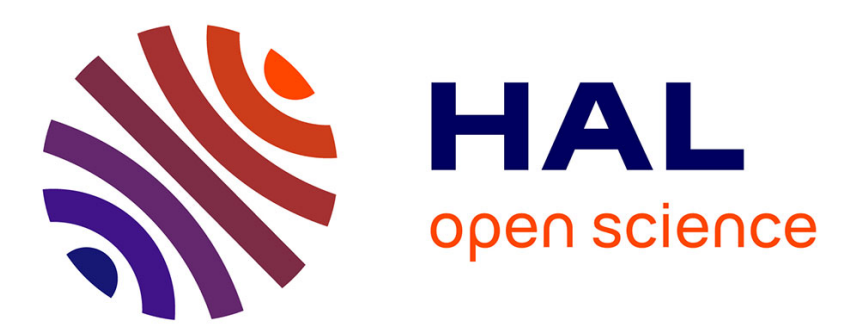

\title{
Stability of the lattice kinetic scheme and choice of the free relaxation parameter
}

Seyed Ali Hosseini, C. Coreixas, N. Darabiha, D. Thevenin

\section{To cite this version:}

Seyed Ali Hosseini, C. Coreixas, N. Darabiha, D. Thevenin. Stability of the lattice kinetic scheme and choice of the free relaxation parameter. Physical Review E , 2019, 99 (6), 10.1103/PhysRevE.99.063305 . hal-02154781

\section{HAL Id: hal-02154781 \\ https://hal.science/hal-02154781}

Submitted on 13 Jun 2019

HAL is a multi-disciplinary open access archive for the deposit and dissemination of scientific research documents, whether they are published or not. The documents may come from teaching and research institutions in France or abroad, or from public or private research centers.
L'archive ouverte pluridisciplinaire HAL, est destinée au dépôt et à la diffusion de documents scientifiques de niveau recherche, publiés ou non, émanant des établissements d'enseignement et de recherche français ou étrangers, des laboratoires publics ou privés. 


\title{
Stability of the lattice kinetic scheme and choice of the free relaxation parameter
}

\author{
S.A. Hosseini, ${ }^{1,2,3}$ C. Coreixas, ${ }^{4}$ N. Darabiha, ${ }^{2}$ and D. Thévenin ${ }^{1}$ \\ ${ }^{1}$ Laboratory of Fluid Dynamics and Technical Flows, \\ University of Magdeburg "Otto von Guericke", D-39106 Magdeburg, Germany \\ ${ }^{2}$ Laboratoire EM2C, CNRS, CentraleSupélec, Université Paris-Saclay, \\ 3 rue Joliot Curie, 91192, Gif-sur-Yvette Cedex, France \\ ${ }^{3}$ International Max Planck Research School (IMPRS) for Advanced \\ Methods in Process and Systems Engineering, Magdeburg, Germany \\ ${ }^{4}$ University of Geneva, Geneva, Switzerland
}

(Dated: June 13, 2019)

\begin{abstract}
The lattice kinetic scheme (LKS), a modified version of the classical single relaxation time (SRT) lattice Boltzmann (LB) method, was initially developed as a suitable numerical approach for nonNewtonian flow simulations and a way to reduce memory consumption of the original SRT approach. The better performances observed for non-Newtonian flows are mainly due to the additional degree of freedom allowing an independent control over the relaxation of higher-order moments, independently from the fluid viscosity. Although widely applied to fluid flow simulations, yet no theoretical analysis of LKS has been performed. The present work focuses on a systematic von Neumann analysis of the linearized collision operator. Thanks to this analysis, the effect of the modified collision operator on the stability domain and spectral behavior of the scheme are clarified. Results obtained in this study show that correct choices of the "second relaxation coefficient" lead, to a certain extent, to more consistent dispersion and dissipation for large values of the first relaxation coefficient. Furthermore, appropriate values of this parameter can lead to a larger linear stability domain. At moderate and low values of the viscosity, larger values of the free parameter are observed to increase dissipation of kinetic modes, while leaving the acoustic modes untouched and having a less pronounced effect on the convective mode. This increased dissipation leads in general to less pronounced sources of non-linear instability, thus improving the stability of the LKS.
\end{abstract}

PACS numbers: 47.11.-j

Keywords: lattice Boltzmann; lattice kinetic scheme; turbulent flows; computational fluid dynamics

\section{INTRODUCTION}

The lattice Boltzmann method, due to its simplicity and efficiency on parallel clusters ${ }^{1}$, has become a numerical tool of choice for computational fluid dynamics. Throughout the past three decades, it has been extended to a wide range of applications from simple incompressible flows to more complex configurations involving multiple phases, compressibility, heat transfer and multiple species 28. In line with the many applications of this scheme, several modified versions of the original formulation have been presented in the literature ${ }^{9}$. The majority of these modified LB schemes were developed and proposed with the aim of extending the stability domain of the solver in order to access higher Reynolds and/or Mach numbers at reasonable costs. They include (but are not limited to) approaches such as the entropic lattice Boltzmann method (ELBM) $)^{10} \mid 18$, the multiple relaxation (MRT) 19 31 , the central moments (CM) or cascaded LBM ${ }^{32} \sqrt[39]{3}$, the regularized (RLBM) ${ }^{40 \mid 50}$ and the Cumulant method 51 54. In addition to these collision models, several numerical discretizations have been used to numerically solve the lattice Boltzamnn equation, see for example $e^{55} \frac{66}{66}$ among many other works.

The LKS, initially developed as a way to reduce memory consumption in LBM ${ }^{67}$, is a modified version of the SRT collision operator which allows for an additional relaxation coefficient (called second relaxation coefficient throughout this manuscript) for higher-order kinetic moments. This approach has mostly been applied to cases involving large variations in the diffusion coefficient (viscosity for flow solvers), such as non-Newtonian flows 68 70, multi-phase flows ${ }^{71,74}$, thermal flows ${ }^{75}$ and viscous fingering in porous media $26 / 77$. In all these examples the viscosity (or species and thermal diffusion coefficient) spans a rather large interval of values. As such the first relaxation coefficient (the only one in the SRT method) might take on values well above 1 , which usually leads to non-negligible errors and unstable simulations. This is mainly due to the fact that for large values of the first relaxation coefficient, higher order terms in the macroscopic PDE recovered by the LB method dominate over terms of orders $\epsilon$ and $\epsilon^{2}$, also referred to as the Euler and Navier-Stokes level terms 50178 . The studies and results published in past studier ${ }^{78}$, showed that the LKS can be perceived as an efficient way to postpone the onset of these higher order moments effects. Although widely used, and already extended to advection-diffusion solvers 79 82, no formal analysis of the operation mode of this collision operator along with the effect of the second relaxation coefficient has been provided in the literature. Only general guidelines concerning the limits of this parameter have been provided ${ }^{83}$, and the stability of the scheme in the case of the advection-diffusion equation has been presented 84 .

Following a previous work where an asymptotic anal- 
ysis of LKS (comprising second and third-order) was presented $\frac{78}{\text {, }}$, this work aims at providing a detailed analysis of the behavior of the scheme at non-vanishing wavenumbers. To that effect, the linearized collision operator is studied using the von Neumann analysis method $20136 / 50|85| 90$. The von Neumann analysis is first used to assess the stability domain of the SRT operator, with equilibrium distribution functions (EDF) of orders two, three and four 50 . Then, the same analysis is applied to the LKS operator for different values of the second relaxation coefficient. The maximum accessible Mach number with the LKS for various values of the viscosity along with the corresponding optimal choices of the second relaxation coefficient are finally found. To get further insights into the operation mode of the LKS, the effects of the second relaxation coefficient on the dispersion and dissipation curves are also systematically studied. The results obtained through the spectral studies are corroborated by simulations of canonical test-cases such as the doubly periodic shear layer and the Taylor-Green vortex.

\section{NUMERICAL SCHEMES}

\section{A. Single relaxation time BGK}

For both numerical methods studied in this work (SRT and LKS), the discrete time evolution of populations $f_{\alpha}$, with a velocity $\boldsymbol{c}_{\alpha}$ and located at $(\boldsymbol{x}, t)$, is defined as:

$$
f_{\alpha}\left(\boldsymbol{x}+\boldsymbol{c}_{\alpha} \delta_{t}, t+\delta_{t}\right)-f_{\alpha}(\boldsymbol{x}, t)=\Omega_{\alpha}(\boldsymbol{x}, t)
$$

where (for the SRT model) the collision operator, $\Omega_{\alpha}$, is the discrete BGK operator:

$$
\Omega_{\alpha}(\boldsymbol{x}, t)=-\frac{1}{\tau}\left(f_{\alpha}-f_{\alpha}^{(e q)}\right)(\boldsymbol{x}, t),
$$

with $\tau$ the discrete relaxation coefficient (for the space and time-discretized Boltzmann equation). The latter is related to the kinematic viscosity $\nu$ through

$$
\tau=\frac{\nu}{c_{s}^{2} \delta_{t}}+\frac{1}{2}
$$

with $c_{s}$ being the isothermal (Newtonian) speed of sound whose dimensionless value is $c_{s} \delta_{t} / \delta_{x}=1 / \sqrt{3}$ for lattices of interest (D2Q9 and D3Q27), and where $\delta_{t}$ and $\delta_{x}$ denote the time-step and grid sizes respectively. The discrete $\operatorname{EDF} f_{\alpha}^{(e q)}$ is defined as an $n^{\text {th }}$ order truncated series of Hermite polynomials 91 :

$$
f_{\alpha}^{(e q), n}=w_{\alpha} \sum_{m=0}^{n} \frac{1}{m ! c_{s}^{2 i}} \boldsymbol{a}_{m}^{(e q)}: \mathcal{H}_{m, \alpha},
$$

where $\boldsymbol{a}_{m}^{(e q)}$ and $\boldsymbol{H}_{m, \alpha}$ are respectively the $i^{\text {th }}$ order Hermite polynomial and coefficient tensors (of rank $m$ ), $w_{\alpha}$ is the weight associated to the discrete velocity $\boldsymbol{c}_{\boldsymbol{\alpha}}$ as obtained from the Gauss-Hermite quadrature, and ":" represents the Frobenius inner tensor product. In the context of the present study, EDFs of order two, three and four will be considered. The corresponding isothermal coefficients are given as:

$$
\begin{gathered}
a_{0}^{(e q)}=\rho, \\
a_{1, i}^{(e q)}=\rho u_{i}, \\
a_{2, i j}^{(e q)}=\rho u_{i} u_{j}, \\
a_{3, i j k}^{(e q)}=\rho u_{i} u_{j} u_{k}, \\
a_{4, i j k l}^{(e q)}=\rho u_{i} u_{j} u_{k} u_{l},
\end{gathered}
$$

and the Hermite polynomial tensors are defined as:

$$
\begin{aligned}
& \mathcal{H}_{0, \alpha}=1 \\
& \mathcal{H}_{1, \alpha, i}=c_{\alpha, i} \\
& \mathcal{H}_{2, \alpha, i j}=c_{\alpha, i} c_{\alpha, j}-c_{s}^{2} \delta_{i j} \\
& \mathcal{H}_{3, \alpha, i j k}=c_{\alpha, i} c_{\alpha, j} c_{\alpha, k} \\
&-c_{s}^{2}\left(c_{\alpha, k} \delta_{i j}+c_{\alpha, j} \delta_{i k}+c_{\alpha, i} \delta_{j k}\right) \\
& \mathcal{H}_{4, \alpha, i j k l}=c_{\alpha, i} c_{\alpha, j} c_{\alpha, k} c_{\alpha, l} \\
&+c_{s}^{4}\left(\delta_{i j} \delta_{k l}+\delta_{i k} \delta_{j l}+\delta_{j k} \delta_{i j}\right) \\
&-c_{s}^{2}\left(c_{\alpha, k} c_{\alpha, l} \delta_{i j}+c_{\alpha, j} c_{\alpha, l} \delta_{i k}+c_{\alpha, j} c_{\alpha, k} \delta_{i l}\right. \\
&\left.+c_{\alpha, i} c_{\alpha, l} \delta_{j k}+c_{\alpha, i} c_{\alpha, k} \delta_{j l}+c_{\alpha, i} c_{\alpha, j} \delta_{k l}\right) .
\end{aligned}
$$

The macroscopic moments, $\rho$ and $u_{i}$, are computed as:

$$
\rho=\sum_{\alpha} f_{\alpha}
$$

$$
\rho u_{i}=\sum_{\alpha} c_{\alpha, i} f_{\alpha}
$$

\section{B. Lattice kinetic scheme}

The LKS collision operator is similar to that of the SRT:

$$
\Omega_{\alpha}=-\frac{1}{\lambda}\left(f_{\alpha}-f_{\alpha}^{(e q, \mathrm{LKS})}\right),
$$

where the so-called second relaxation coefficient $\lambda$ relates to the fluid kinematic viscosity $\nu$ as:

$$
\lambda-A=\tau=\frac{\nu}{c_{s}^{2} \delta_{t}}+\frac{1}{2} .
$$

$\lambda$ can be seen as a free parameter where $A$ is a constant fixed by the choice of the free parameter, while $\lambda-A$ is fixed by the kinematic fluid viscosity. For the sake of clarity, in the remainder of this manuscript we will refer to a second viscosity in the model, denoted $\eta$, which is directly related to the free parameter and defined as:

$$
\lambda=\frac{\eta}{c_{s}^{2} \delta_{t}}+\frac{1}{2} .
$$


This second viscosity $\eta$ is not a physical parameter and especially not to be confused with the fluid bulk viscosity (designated by $\zeta$, equal to $\frac{2}{3} \mu$ for a monatomic gas, $\mu$ being the dynamic viscosity, in the SRT 3D model, and left unaffected by the modified collision operator in LKS ${ }^{78}$ ). The equilibrium distribution function is then defined as:

$$
\begin{aligned}
f_{\alpha}^{(e q), \mathrm{LKS}}= & f_{\alpha}^{(e q), n}+\frac{A}{2 c_{s}^{4}(A-\lambda)} \boldsymbol{\Pi}^{(n e q)}: \mathcal{H}_{2, \alpha} \\
= & w_{\alpha} \sum_{m=0}^{n} \frac{1}{m ! c_{s}^{2 m}} \boldsymbol{a}_{m}^{(e q)}: \mathcal{H}_{m, \alpha} \\
& +\frac{w_{\alpha} A}{2 c_{s}^{4}(A-\lambda)} \boldsymbol{\Pi}^{(n e q)}: \mathcal{H}_{2, \alpha},
\end{aligned}
$$

where in addition to the SRT equilibrium state $f_{\alpha}^{(e q), n}$ (at order $n$ ), we have a contribution proportional to the second-order non-equilibrium moment:

$$
\boldsymbol{\Pi}^{(n e q)}=\boldsymbol{\Pi}-\boldsymbol{\Pi}^{(e q)}=\sum_{\alpha} \boldsymbol{c}_{\alpha} \otimes \boldsymbol{c}_{\alpha}\left(f_{\alpha}-f_{\alpha}^{(e q)}\right) .
$$

Interestingly, thanks to Eq. (9), and using the conservation of mass

$$
\boldsymbol{a}_{0}^{(n e q)}=0
$$

one can rewrite Eq. 11a using Hermite coefficients

$$
f_{\alpha}^{(e q), \mathrm{LKS}}=f_{\alpha}^{(e q), n}-\frac{A}{\tau} \frac{w_{\alpha}}{2 c_{s}^{4}} \boldsymbol{a}_{2}^{(n e q)}: \mathcal{H}_{2, \alpha} .
$$

This is the starting point to link the LKS with the original regularized collision mode ${ }^{40[41}$, as demonstrated in App. A.

\section{VON NEUMANN STABILITY}

In this section, through the use of the von Neumann linear stability analysis method, the stability of the LKS and SRT collision operators are assessed. Furthermore the effects of the free parameter on the stability and dispersion-dissipation properties of the LKS are studied.

\section{A. Von Neumann stability analysis formalism}

In the present work, the von Neumann (VN) stability analysis aims at studying the time evolution of a perturbation $f_{\alpha}^{\prime}$ that is injected into the lattice Boltzmann equation after its time and space discretization. This perturbation is assumed to have the form of a monochromatic plane wave, whose propagation speed and attenuation rate will be obtained as a result of the $\mathrm{VN}$ analysis. If the resulting attenuation rate is positive then the amplitude of the perturbation will grow over time. This will eventually result in an unstable numerical scheme for the set of parameters considered ( $\tau$, Ma, etc). On the contrary, the LBM will be linearly stable if it remains negative.

As a consequence, the VN stability analysis can be used to evaluate the linear stability domain of a LBM for a given set of parameters in an a priori manner. More specifically, the VN analysis can be used to $o b$ jectively evaluate the stabilization properties of a particular collision model as long as its assumptions (small amplitude of the perturbation, periodic domain, uniform grid) remain valid. It has been widely used in the past years to evaluate the stability properties of the lattice Boltzmann method. Interested readers are referred tc 20|36|86 90|94|100, among other sources.

Starting with a Taylor-McLaurin expansion around a mean flow state, $\bar{f}_{\alpha}$ :

$$
f_{\alpha} \approx \bar{f}_{\alpha}+f_{\alpha}^{\prime}
$$

$$
\left.\Omega_{\alpha}\left(f_{\alpha}\right) \approx \Omega_{\alpha}\right|_{\bar{f}_{\alpha}}+J_{\alpha \beta} f_{\beta}^{\prime},
$$

where Einstein's summation rule over $\beta$ is used. In addition, $J_{\alpha \beta}$ is the Jacobian of the collision operator evaluated about $\bar{f}_{\beta}$, i.e,

$$
J_{\alpha \beta}=\left.\partial_{f_{\beta}} \Omega_{\alpha}\right|_{\bar{f}_{\beta}} .
$$

Placing these expressions into the discrete LB timeevolution equation:

$$
\begin{array}{r}
f_{\alpha}^{\prime}\left(\boldsymbol{x}+\boldsymbol{c}_{\alpha} \delta_{t}, t+\delta_{t}\right)-f_{\alpha}^{\prime}(\boldsymbol{x}, t)=J_{\alpha \beta} f_{\alpha}^{\prime}(\boldsymbol{x}, t) \\
-\underbrace{\left(\bar{f}_{\alpha}\left(\boldsymbol{x}+\boldsymbol{c}_{\alpha} \delta_{t}, t+\delta_{t}\right)-\bar{f}_{\alpha}(\boldsymbol{x}, t)-\left.\Omega_{\alpha}\right|_{\bar{f}_{\alpha}}\right)}_{=0},
\end{array}
$$

and taking out the last terms on the RHS one gets:

$$
f_{\alpha}^{\prime}\left(\boldsymbol{x}+\boldsymbol{c}_{\alpha} \delta_{t}, t+\delta_{t}\right)=\left(\delta_{\alpha \beta}+J_{\alpha \beta}\right) f_{\beta}^{\prime}(\boldsymbol{x}, t),
$$

where $\delta_{\alpha \beta}$ is the Kronecker delta function. Using the SRT collision operator, we can then re-write the linearized time-evolution equation as:

$$
\begin{aligned}
f_{\alpha}^{\prime}\left(\boldsymbol{x}+\boldsymbol{c}_{\alpha} \delta_{t}, t+\delta_{t}\right)= & \\
& {\left[\left(1-\frac{1}{\tau}\right) \delta_{\alpha \beta}+\frac{1}{\tau} J_{\alpha \beta}^{(e q), n}\right] f_{\beta}^{\prime}(\boldsymbol{x}, t), }
\end{aligned}
$$

with $J_{\alpha \beta}^{(e q), n}=\left.\partial_{\beta} f_{\alpha}^{(e q), n}\right|_{\bar{f}_{\beta}}$ and $\bar{f}_{\beta}=f_{\beta}^{(e q), n}(\bar{\rho}, \overline{\boldsymbol{u}})$. To compute the Jacobian matrix of the second-order EDF, knowing that $\partial_{f_{\beta}} f_{\gamma}=\delta_{\beta, \gamma}$, the following expressions can be used:

$$
\begin{gathered}
\partial_{f_{\beta}} \boldsymbol{a}_{0}^{(e q)}=\partial_{f_{\beta}}(\rho)=\sum_{\gamma} \delta_{\beta, \gamma}=1, \\
\partial_{f_{\beta}} \boldsymbol{a}_{1}^{(e q)}=\partial_{f_{\beta}}(\rho \boldsymbol{u})=\sum_{\gamma} \boldsymbol{c}_{\gamma} \delta_{\beta, \gamma}=\boldsymbol{c}_{\beta},
\end{gathered}
$$




$$
\begin{array}{r}
\partial_{f_{\beta}} \boldsymbol{a}_{2}^{(e q)}=\partial_{f_{\beta}}(\rho \boldsymbol{u} \otimes \boldsymbol{u})=-\frac{\boldsymbol{a}_{1}^{(e q)} \otimes \boldsymbol{a}_{1}^{(e q)}}{\left(\boldsymbol{a}_{0}^{(e q)}\right)^{2}} \\
+\frac{\boldsymbol{a}_{1}^{(e q)} \otimes \boldsymbol{c}_{\beta}+\left(\boldsymbol{a}_{1}^{(e q)} \otimes \boldsymbol{c}_{\beta}\right)^{T}}{\boldsymbol{a}_{0}^{(e q)}} .
\end{array}
$$

Eventually, the Jacobian matrix of the second-order EDF reads as

$$
J_{\alpha \beta}^{(e q), 2}=w_{\alpha}\left(\mathcal{H}_{0, \alpha}+\mathcal{H}_{1, \alpha}: \frac{\boldsymbol{c}_{\beta}}{c_{s}^{2}}+\mathcal{H}_{2, \alpha}: \frac{\partial_{f_{\beta}} \boldsymbol{a}_{2}^{(e q)}}{2 c_{s}^{4}}\right) .
$$

The extension of this development to higher order EDFs (i.e. third and fourth order) is given in Appendix B.

Following the previously-derived equations, the additional term within the EDF of the LKS can be rewritten thanks to:

$$
\partial_{f_{\beta}} \boldsymbol{a}_{2}^{(n e q)}=\mathcal{H}_{2, \beta}-\partial_{f_{\beta}} \boldsymbol{a}_{2}^{(e q)}
$$

so that

$$
J_{\alpha \beta}^{(e q), \mathrm{LKS}}=J_{\alpha \beta}^{(e q), n}-\frac{A}{\tau} \frac{w_{\alpha}}{2 c_{s}^{4}} \mathcal{H}_{2, \alpha}:\left(\mathcal{H}_{2, \beta}-\partial_{f_{\beta}} \boldsymbol{a}_{2}^{(e q)}\right) .
$$

The last step of the $\mathrm{VN}$ analysis is to assume that perturbations $f_{\alpha}^{\prime}$ are monochromatic plane waves :

$$
f_{\alpha}^{\prime}=F_{\alpha} \exp [j(\boldsymbol{k} \cdot \boldsymbol{x}-\omega t)]
$$

where $F_{\alpha}$ is the wave amplitude, $j$ is the imaginary unit, $\|\boldsymbol{k}\|=k$ is the wave number, and $\omega$ is the complex time frequency of the wave. $k$ is related to the wave length of $f_{\alpha}^{\prime}$, whereas $\Im(\omega)$ and $\Re(\omega)$ are related to its attenuation and propagation speed (dissipation and dispersion, studied in details in the next sections). By injecting these perturbations into Eq. (17) one obtains the following eigenvalue problem of size $V$ (the number of discrete velocities) :

$$
\boldsymbol{M} \boldsymbol{F}=\exp (-j \omega) \boldsymbol{F}
$$

$\boldsymbol{F}$ is the eigenvector composed of all amplitudes. It is tightly related to the eigenvalue $\exp (-j \omega) . \quad \boldsymbol{M}$ is the matrix associated to Eq. (17). In the present work, this matrix can be expressed as :

$$
\boldsymbol{M}=\boldsymbol{E}[\boldsymbol{\delta}+\boldsymbol{J}]
$$

with

$$
E_{\alpha \beta}=\exp \left[-j\left(\boldsymbol{c}_{\alpha} \cdot \boldsymbol{k}\right)\right] \delta_{\alpha \beta}
$$

For the SRT collision mode, the Jacobian matrix $\boldsymbol{J}$ is defined as :

$$
J_{\alpha \beta}^{\mathrm{SRT}, n}=-\frac{1}{\tau}\left(\delta_{\alpha \beta}-J_{\alpha \beta}^{(e q), n}\right)
$$

whereas for the LKS, we end up with

$$
J_{\alpha \beta}^{\mathrm{LKS}}=-\frac{1}{\lambda}\left(\delta_{\alpha \beta}-J_{\alpha \beta}^{(e q), \mathrm{LKS}}\right) .
$$

It is important to notice that the matrix $\boldsymbol{M}$, and a fortiori the eigenvalue problem (26) itself, depend on: (1) the mean flow $(\bar{\rho}, \overline{\boldsymbol{u}}),(2)$ the wave number $\left(k_{x}\right.$ and $k_{y}$ in $2 \mathrm{D}$ ), and (3) the relaxation coefficient $\tau$ or equivalently the kinematic viscosity $\nu$. This means that for each set of these parameters the eigenvalue problem needs to be solved to obtain the corresponding values of $\Re(\omega)$ and $\Im(\omega)$. Doing so, the spectral properties (dispersion and dissipation) can be obtained for any given collision model. In the particular case of the LKS, a second relaxation parameter $\lambda$ is used to postpone the onset of the high-order moments belonging to the lattice Boltzmann dynamics. By solving its related eigenvalue problem, it is intended in the present work to further quantify the impact of this second relaxation parameter, and improve the spectral properties of the LKS in a consistent way.

\section{B. Linear stability domain}

The classical SRT-BGK operator with a second-order EDF in the context of the LB method is known to have a very restricted stability domain (in terms of the maximum Mach number) for small values of the nondimensional viscosity. Furthermore, various studies have shown that including higher-order terms of the Hermite expansion in the EDF can have a non-negligible effect on the stability domain $50 / 101$.

As a first investigation, the stability areas of the different order EDFs with the SRT collision operator are illustrated in Fig. 1 for very large variations of the dimensionless kinematic viscosity $\nu \delta_{t} / \delta_{x}^{2}$. The stability plot

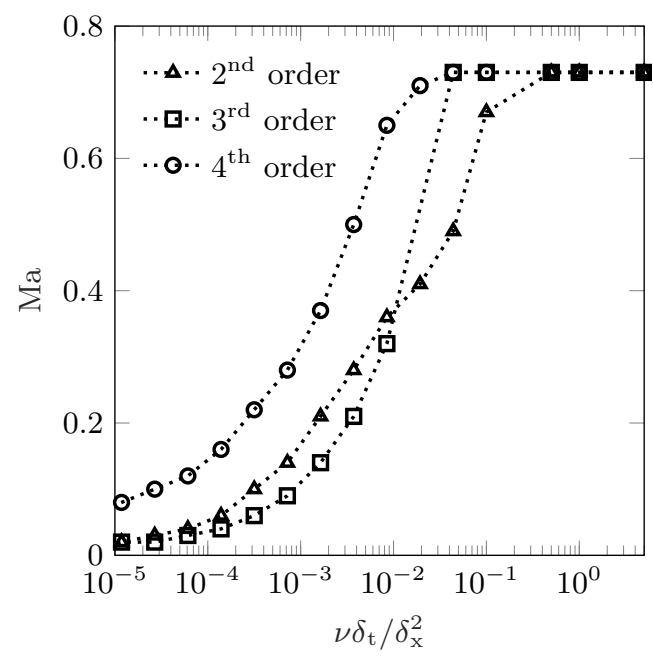

FIG. 1: Effects of the EDF order on the linear stability domain of the SRT collision model. 
are defined following the protocol described in a previous study 101 , which consists in computing the maximal Mach number for which the growth rate $\Im(\omega)$ remains negative over the entire upper side of the Fourier space, i.e., $\left[k_{x}, k_{y}\right] \in[-\pi, \pi] \times[0, \pi]$. This interval is spaced with $\Delta k=0.02$, and the orientation of the mean flow (with respect to the $x$-axis) goes from 0 to $\pi / 4$ (steps of $\pi / 45$ ). The eigenvalue problem has been solved for a non-dimensional viscosity taking on the following 12 values:

$$
\nu \delta_{t} / \delta_{x}^{2}=\left\{1 \times 10^{p}, 5 \times 10^{p}\right\}, \quad p \in \llbracket-5,0 \rrbracket .
$$

As shown in Fig. 1, the maximum allowed Mach number drastically decreases for non-dimensional viscosities below $10^{-2}$. Furthermore, it can be seen that while using a third-order EDF does not improve noticeably the stability behavior, the use of a fourth-order EDF has a non-negligible effect on the stability domain. This is counter-intuitive since the use of third-order terms helps reducing the number of error terms $\left(\mathcal{O}\left(\mathrm{Ma}^{3}\right)\right)$ in the viscous stress tensor, whereas the fourth-order term should not have any impact at the leading order on the macroscopic behavior of the LBM ${ }^{50 \mid 101}$. Knowing this, one may wonder if alternative approaches to deriving the discrete EDF might allow us to further improve the linear stability of the SRT-LBM. In particular, equilibria obtained through the principle of maximum entropy $\frac{12102}{104}$ have not been studied from the LSA viewpoint yet. Such a study is out of the scope of the present work, but results concerning their impact on the stability of the SRT-LBM will definitely be discussed in a future work.

As discussed in the introduction, and based on high order asymptotic analyses presented previously ${ }^{78}$ along with numerical examples, the free parameter in the LKS is expected to have an effect on the stability domain, as it provides independent control over the relaxation of higher-order moments. To that end, the effects of this parameter have been evaluated through a systematic von Neumann analysis. In these studies, the second non-dimensional viscosity related to the relaxation of higher-order moments takes on the same values as the first non-dimensional viscosity, resulting in a total of 144 studied configurations. The obtained results for EDFs of orders two, three and four along with the corresponding stability maps are displayed in Figs. 2 and 3.

In all three cases, the additional degree of freedom provided by the LKS collision operator has a huge impact on the stability domain of the LBM. As compared to the SRT collision model $(\eta=\nu)$, the stability area drastically decreases for large values of the non-dimensional viscosity $\left(\nu \delta_{t} / \delta_{x}^{2} \gtrsim 10^{-2}\right)$ when the second parameter is fixed to very low values $\left(\eta \delta_{t} / \delta_{x}^{2} \lesssim 10^{-4}\right)$. Furthermore, the opposite behavior is observed when $\eta \delta_{t} / \delta_{x}^{2}$ is fixed to values greater than $10^{-2}$, while $\nu \delta_{t} / \delta_{x}^{2} \lesssim 10^{-4}$. When it comes to the impact of the EDF order, one can notice that the third-order EDF does not really help improving the linear stability of the LKS, except for very large values of both dimensionless parameters. On the contrary, the fourth-order EDF drastically improves the moderate to low viscosity regime $\left(\nu \delta_{t} / \delta_{x}^{2} \lesssim 10^{-3}\right)$ for most values of the second parameters $\left(\eta \delta_{t} / \delta_{x}^{2} \lesssim 1\right)$; this is in agreement with the results obtained previously for the SRT operator. The effects of both EDF order and the LKS collision operator are better shown in Fig. 4. Although a major issue, linear stability does not guarantee an optimal behavior for the numerical scheme, especially for under-resolved features where most numerical schemes are known to exhibit non-physical velocities at higher wavenumbers. To better qualify the behavior of the SRT collision operator and the effect of the LKS, the dissipation-dispersion behavior of the convective mode are studied in the next subsections.

\section{Dispersion-dissipation behavior}

Through the linear stability analysis presented in the previous section, it has been established that for both LKS and SRT, the discrete time-evolution operators might be stable for relatively high Mach numbers in this range of physical viscosity if the second parameter $\lambda$ is properly adjusted. However, the numerical stability along with the asymptotic analysis do not necessarily guarantee the recovery of the correct flow physics for any grid configuration; they only guarantee the convergence of the numerical scheme towards the continuous solution in the limit of vanishing wavenumbers.

The present subsection will focus on clarifying the effect of the free parameter for non-vanishing wavenumbers by looking at the dispersion curves on the $k_{x}$ axis, assuming the background velocity is parallel to this axis (i.e., $\left.k_{y}=0\right)$. Futhermore, only results obtained with fourthorder EDFs will be considered as they yield the largest stability areas (and given that they have been shown to have a beneficial effect on dispersion-dissipation properties $50|90| 101)$.

To better understand the limitations for simulations with large variations in the relaxation coefficient (apart from the stability domain), the dispersion and dissipation of the convective mode in the SRT collision operator for different values of the non-dimensional viscosity and a given Mach number of 0.1 are shown in Fig. 5 . As seen in this figure, while for values of the non-dimensional viscosity up to 0.1 the dispersion and dissipation curves follow closely the asymptotic curves up to $k_{x} \delta_{x} \sim \pi / 2$, for larger values the discrepancies appear much sooner, around $\pi / 8$ for $\nu \delta_{x} / \delta_{t}^{2}=0.5$ and $\pi / 16$ for $\nu \delta_{x} / \delta_{t}^{2}=1$. This reflects the fact that for high values of the kinematic viscosity, or equivalently of the relaxation coefficient, the continuum limit, upon which the Chapman-Enskog assumption relies, is not valid anymore. Consequently, the LBM does not converge towards the correct macroscopic behavior for high values of the kinematic viscosity, even for moderate values of the wave number. One can even show that under these conditions the lattice Boltzmann equation itself does not recover the proper macroscopic 

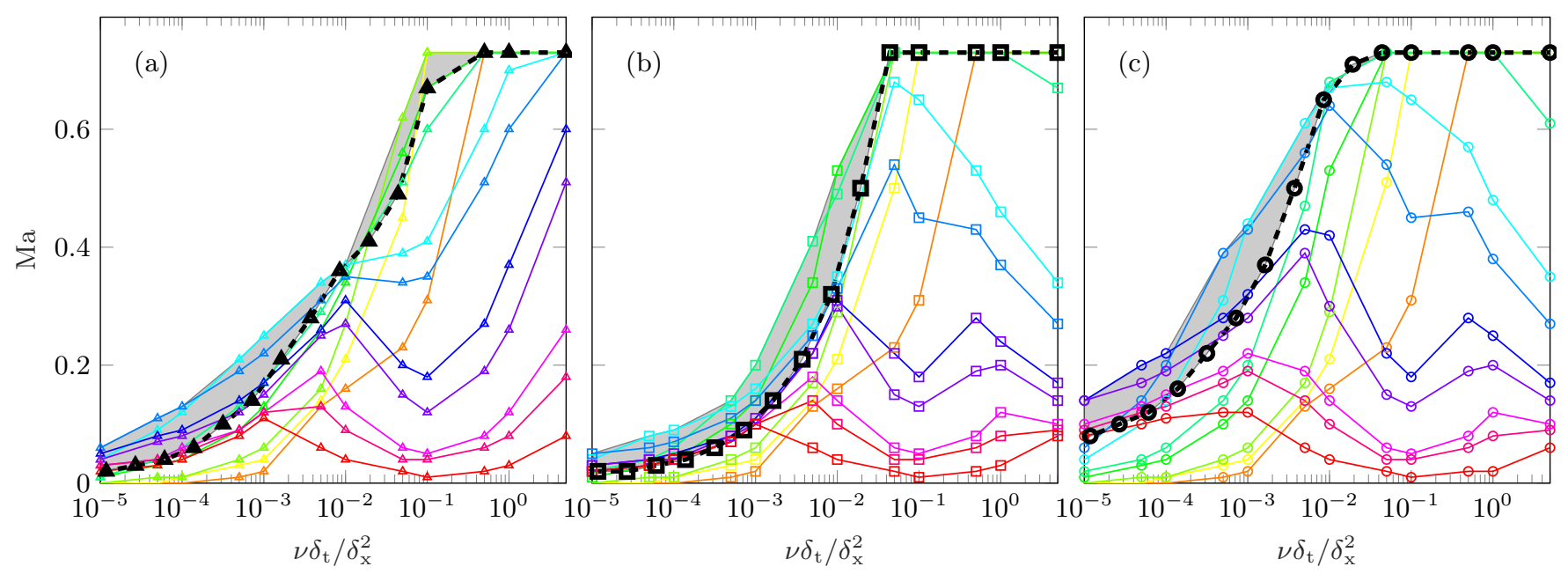

FIG. 2: Stability areas of the SRT (- - -) and LKS with EDFs of (a) second, (b) third and (c) fourth order, for different values of the free parameter: $\eta \delta_{t} / \delta_{x}^{2}=5(-), 1(-), 5 \times 10^{-1}(-), 1 \times 10^{-1}(-), 5 \times 10^{-2}(-)$, $1 \times 10^{-2}(-), 5 \times 10^{-3}(-), 1 \times 10^{-3}(-), 5 \times 10^{-4}(-), 1 \times 10^{-4}(-), 5 \times 10^{-5}(-), 1 \times 10^{-5}(-)$.
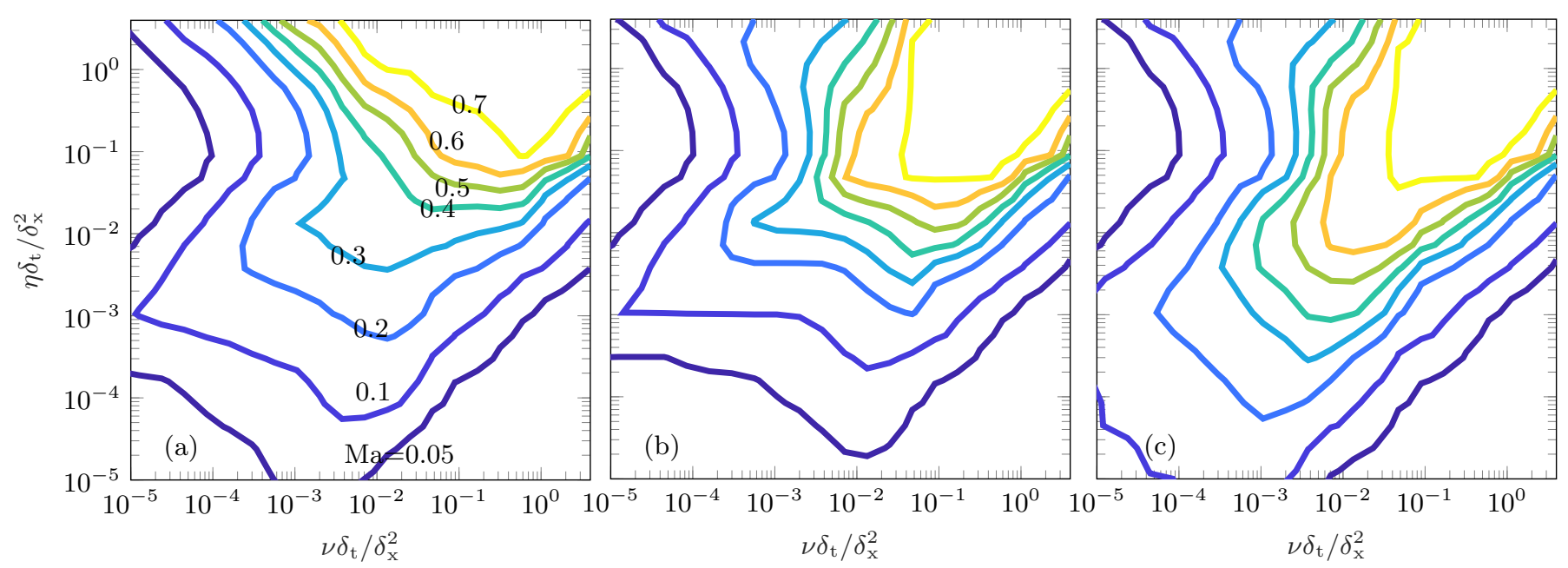

FIG. 3: Stability maps for EDFs of order (a) two, (b) three and (c) four (, color codes are the same for all figures).

behavior for high wave numbers 50 .

From a practical point of view, if one still wants to simulate flows with high values of the kinematic viscosity, then all flow feature must be well resolved (e.g. for $\nu \delta_{x} / \delta_{t}^{2}=1$, smallest features must be resolved by at least 32 grid points) in order for the scheme to correctly capture the physics (i.e., the correct signal propagation speed).

At large first non-dimensional viscosity coefficients and moderate Mach numbers (typically encountered in nonNewtonian flow simulations), Navier-Stokes level dynamics are only correctly recovered for very well-resolved features, as shown previously. To see the effects of the choice of the free parameter in such configurations, dispersiondissipation curves on the $k_{x}$ axis are studied for different combinations of $\nu \delta_{t} / \delta_{x}{ }^{2}$ and $\eta \delta_{t} / \delta_{x}{ }^{2}$. The corresponding results are shown in Fig. 6. Looking at the upper row it is observed that the choice of the free parameter affects the dispersion curve. Referring back to results shown in Fig. 6, it is readily observed that optimal choices of the free parameter can improve the dispersion behavior of the scheme for large values of the non-dimensional relaxation coefficient. For example, setting $\eta \delta_{t} / \delta_{x}{ }^{2}$ to 0.5 in the first cases $\left(\nu \delta_{t} / \delta_{x}{ }^{2}=1\right)$ extends the usable portion of the $k_{x}$ axis from $\pi / 16$ to $\pi / 8$. This would potentially reduce: (1) the number of grid points by a factor of $2^{3}$ for a typical 3-D simulation, and (2) the number of iterations required to simulate a prescribed physical time (whatever the scaling used for the computation of the time step). The same observation also holds for other values of the first non-dimensional viscosity (values leading to first relaxation coefficients above 1). In all cases, in agreement with asymptotic analyses, setting the second viscosity to lower values (lower than the hydrodynamic viscosity) im- 


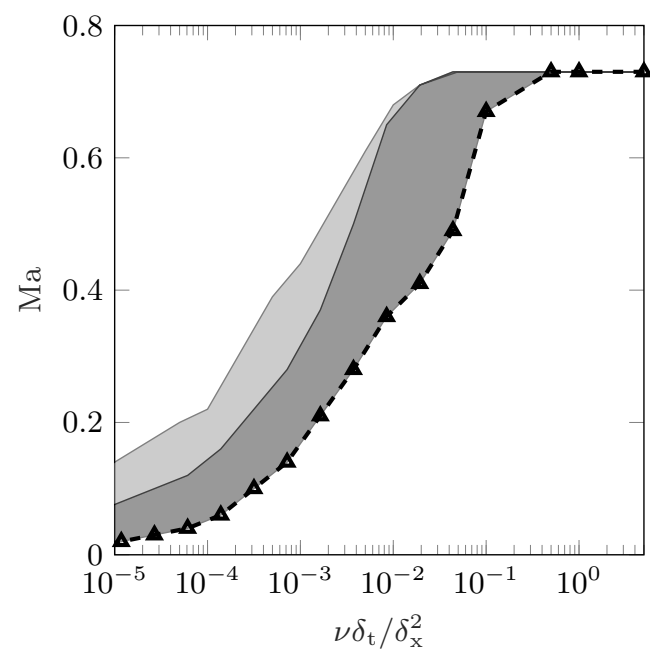

FIG. 4: Effects of the EDF order and of the LKS collision operator on stability domain - the darker gray area represents the gain induced by the order of the EDF (going from second to fourth order), while the lighter gray area corresponds to the LKS collision operator (LKS with fourth-order EDF).

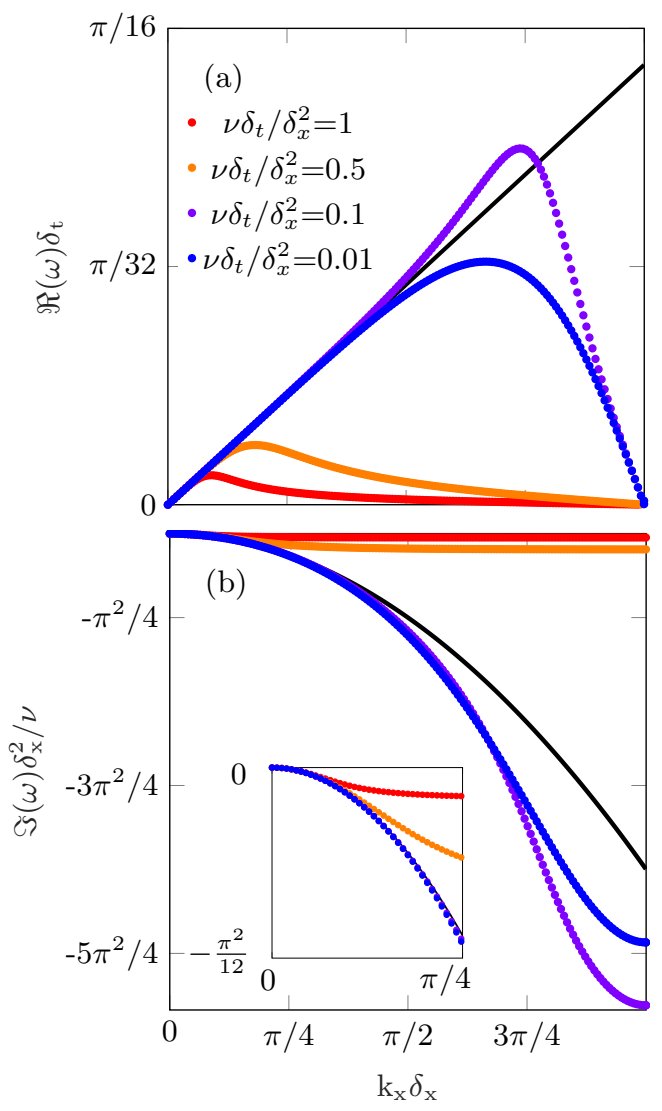

FIG. 5: Effects of the first relaxation coefficient on (a) spectral dispersion and (b) dissipation for the SRT collision operator. proves the spectral behavior of the dispersion curve.

The choice of the free parameter has also an effect on the long wavenumber region of the dispersion curves. For the SRT collision operator, after reaching a maximum, the dispersion curves switch direction and come back down towards the $x$-axis at long wavenumbers. On the contrary, for the LKS, below a certain value for the second viscosity this change of sign of the dispersion curve slope is not observed anymore. This phenomenon is interesting because it coincides with a modal "curve-veering" type interaction in the dissipation curves. While lower values of the free parameter usually lead to higher dissipation at higher wavenumbers, below a certain threshold, due to modal interaction (with a kinetic mode) the convective mode changes direction. This effect can be observed in all three cases shown in Fig. 6. For instance for the leftmost case: for $\eta \delta_{t} / \delta_{x}^{2}=1.0$ and 0.5 the dispersion curve slope changes sign around $\pi / 8$; however, for $\eta \delta_{t} / \delta_{x}^{2}=0.32$ and 0.2 this is not observed anymore. Looking at the corresponding dissipation curve it is readily observed that for the latter cases, the convective mode interacts and collapses with a kinetic mode at around $\pi / 8$, while this interaction is not observed for the other two configurations. In some cases, this modal interaction leads to the convective mode taking on eigenvalues with a positive imaginary part, thus leading to a linearly unstable simulation. The case with $\nu \delta_{t} / \delta_{x}{ }^{2}=0.3$ and $\eta \delta_{t} / \delta_{x}{ }^{2}=0.05$ displayed in the lower leftmost sub-figure in Fig. 6 6 showcases such a behavior. In this subfigure, one can observe that $\eta \delta_{t} / \delta_{x}{ }^{2}=0.05$ leads to lower dissipation above $k_{x}=\pi / 4$ as compared to $\eta \delta_{t} / \delta_{x}{ }^{2}=0.167$; in the former case the convective mode curve interacts with a kinetic mode and as a result its slope changes sign. One can therefore conclude that for such configurations (large non-dimensional viscosities) the free parameter can be chosen to maximize either dissipation of high wavelength features or the wavelength resulting in acceptable deviations of the spectral velocity from the asymptotic one. The latter concept is illustrated in Fig. 7. Choosing the spectral speed error tolerance to be $10 \%$ (dashed black lines in Fig. 7), it is observed that setting $\eta \delta_{t} / \delta_{x}^{2}$ to 0.2 yields the best results for the configuration displayed in Fig. 7, as it extends the range of wavenumbers with coherent propagation velocity from $\pi / 4$ to approximately $\pi / 2$.

On the other end of the spectrum, for vanishing physical non-dimensional viscosities, the choice of the free parameter has very minor effects on the spectral behavior of the dispersion curve. It can, however, be used as a way to maximize dissipation of kinetic modes. A thorough study of the spectral behavior at moderate Mach numbers shows that the dissipation of the acoustic modes is not affected by the choice of the free parameter. The convective mode experiences changes in dissipation centered around $3 \pi / 4$. Larger values of the free parameter lead to lower dissipation around this wavenumber, eventually leading to linear instability above a certain threshold. All kinetic modes experience higher dissipation for 

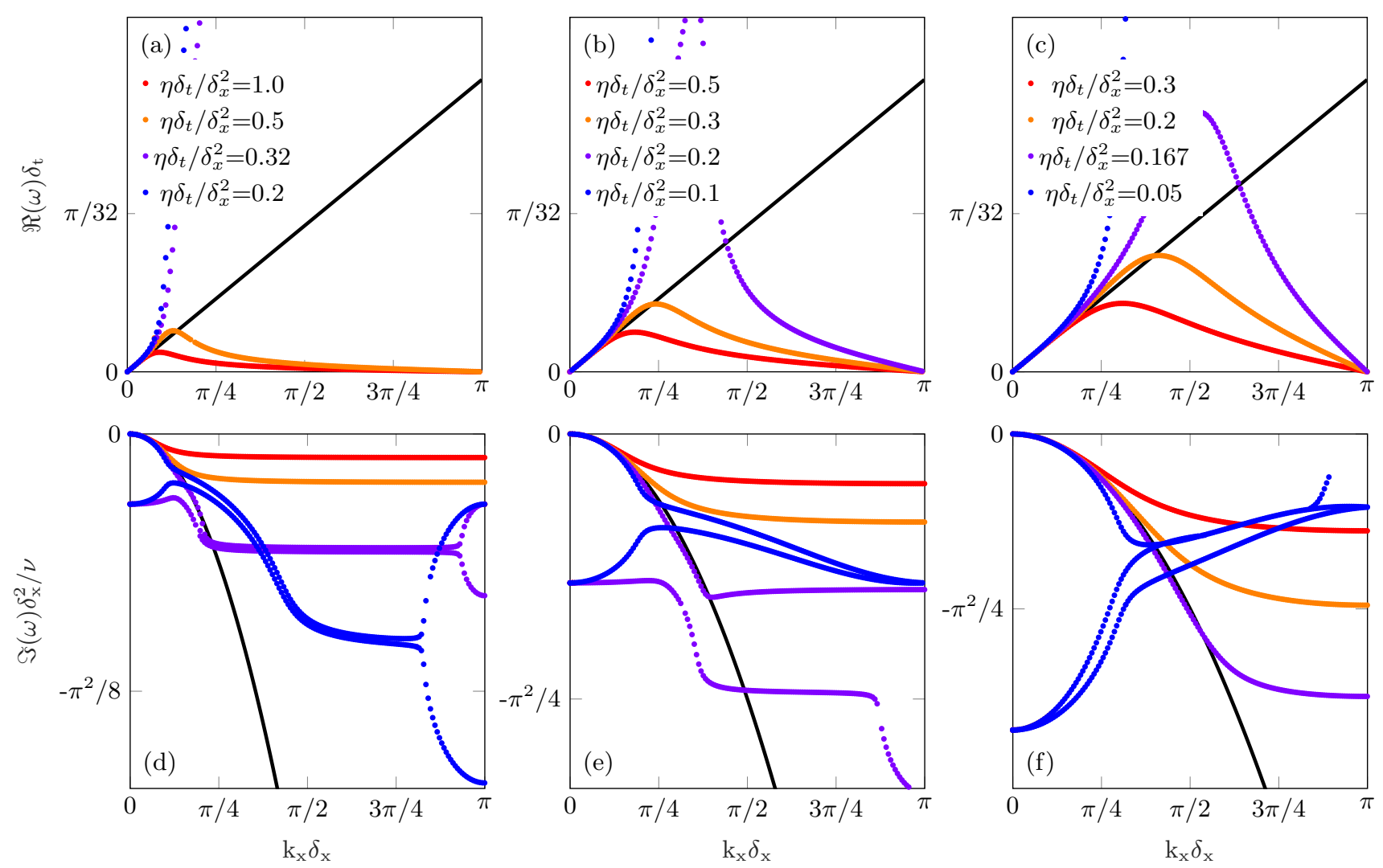

FIG. 6: Effects of the second relaxation coefficient on spectral dispersion ( $a, b$ and c) and dissipation (d, e and f) for large values of non-dimensional viscosity, from left to right: $\nu \delta_{t} / \delta_{x}^{2}=1,0.5,0.3$.

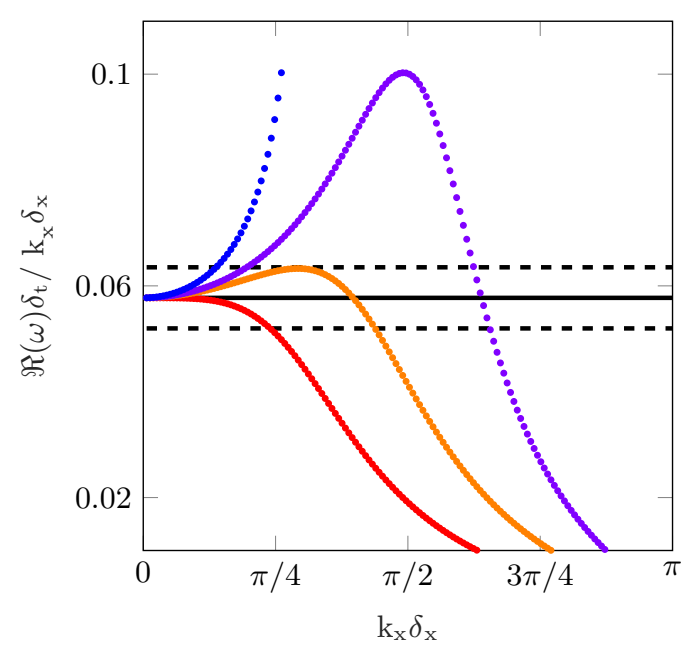

FIG. 7: Spectral velocity of the LKS model with $\nu \delta_{t} / \delta_{x}^{2}=0.3$ and $\mathrm{Ma}=0.1$ for different choices of the free parameter; Choices of the free parameter and the color coding are the same as in the rightmost cases in Fig. 6.

larger values of the free parameter. The interplay between the different modes and the choice of the free parameter for low non-dimensional viscosities and moderate Mach numbers detailed in this paragraph are illustrated in Fig. 8. The stabilizing effect of over-dissipation of kinetic moments has readily been established through the regularized LBM. But the linear stability analysis of the LKS and the corresponding dissipation curves show that at vanishing non-dimensional physical viscosities, setting $\lambda=1$ can lead to reduced linear stability domains. The free parameter can therefore be chosen as the value maximizing dissipation of kinetic modes while resulting in a linearly stable scheme (based on the maximum Mach number in the considered simulation).

As a conclusion, using a thorough linear stability analysis and a study of the dispersion-dissipation curve the following can be established: (a) the stability domain (maximum stable Mach number) as a function of the first and second relaxation coefficient for different orders of the EDF, (b) the effect of the choice of the free parameter on dispersion and dissipation for large values of the non-dimensional viscosity, and (c) the effect of the free parameter on dissipation of the different hydrodynamic and kinetic modes for small values of the nondimensional viscosity. To better understand these effects and the impact of over-relaxation of kinetic modes on sources of non-linear instability the next section will focus on using the LKS for two canonical fluid dynamic test-cases, namely the doubly periodic shear layer and the 3-D Taylor-Green vortex. 

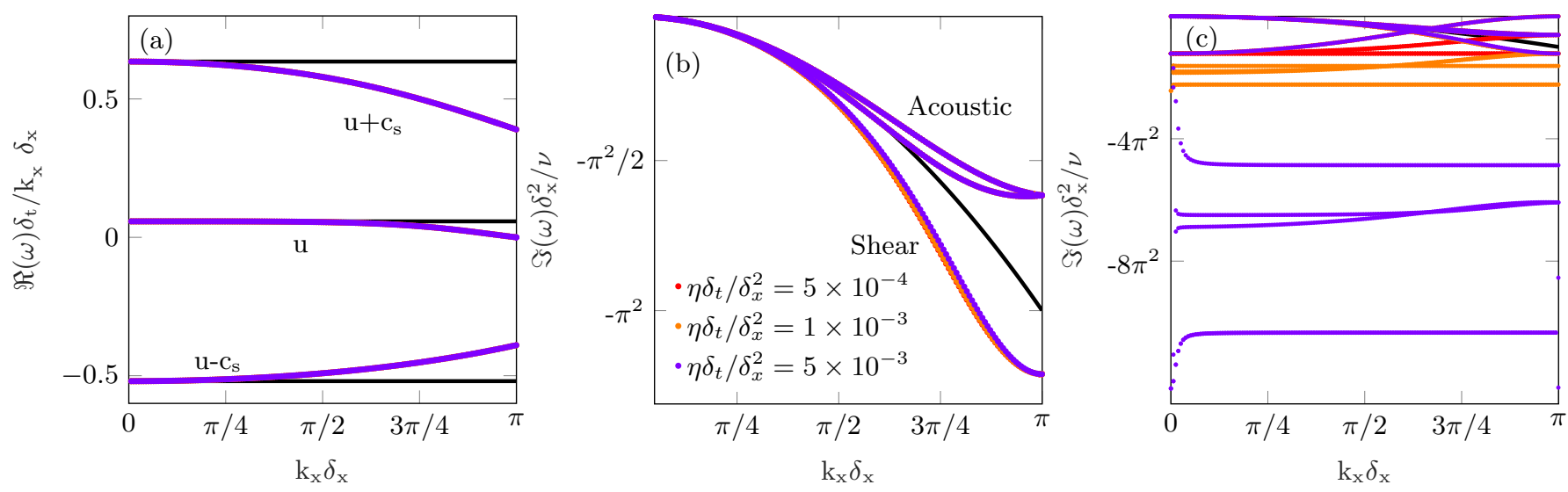

FIG. 8: Effects of the second relaxation coefficient for $\nu \delta_{t} / \delta_{x}^{2}=5 \times 10^{-4}$ and Mach $=0.1$ on: (a) dispersion of acoustic and convective modes, (b) dissipation of acoustic and convective modes, (c) dissipation of hydrodynamic and kinetic modes. The dispersion and dissipation curves of convective and acoustic modes for all values of $\eta \delta_{t} / \delta_{x}^{2}$ can not be clearly distinguished as they exactly fall onto each other.

\section{NUMERICAL SIMULATIONS AND RESULTS}

Throughout this section, only collision models based on the fourth-order EDF will be considered as it led to the widest stability area and seemed to have a beneficial impact on dispersion properties. The effect of the order of the EDF on spectral properties is illustrated in Appendix C Simulations are performed with the D2Q9 (for 2-D) and D3Q27 (for 3-D) stencils. While the double shear layer test-case aims at further evaluating the stability domain of collision models of interest, the simulation of the turbulent Taylor-Green vortex is conducted to investigate the impact of the second relaxation parameter $\lambda$ on higher wavenumbers.

\section{A. Doubly-periodic shear layer : Non-linear effects}

The double shear layer is a well-known test-case used in particular to establish the ability of numerical schemes to cope with unresolved flow features. It was for instance used by Brown \& Minion to assess: (1) the stability of a number of finite-difference schemes, and (2) their ability to deal with under-resolved gradients that induce the generation of spurious Gibbs oscillations $105 / 106$. It can be perceived as a tool to enrich results derived through linear von Neumann analysis, as non-linear effects are readily observed in this test-case. The main numerical artifacts appearing in this test-case (spurious vortices) have also been identified as small wavelength effects resulting from nonlinear truncation error terms of the discretized solvers $105 \mid 106$.

This 2-D flow is made up of two longitudinal shear layers, located at $y=L / 4$ and $y=3 L / 4$, and that evolve in a doubly-periodic simulation domain of size $L \times L$. Periodicity allows to study the stability properties of the bulk solver without any effects from boundary conditions 107 . As a result of a small perturbation introduced in the velocity field (perpendicular to the shear layers), the shear layers roll-up -due to a Kelvin-Helmholtz instability- and eventually generate two counter-rotating vortices. For many numerical schemes, the under-resolved simulations of this test-case result in additional spurious vortices, ultimately leading to divergence (in some cases the additional vortices are effectively dissipated and do not lead to blow-up). Given that the formation of the additional vortices have been identified as non-linear effects, cases leading to numerical blow-ups through these numerical artifacts are interesting to study (especially when instabilities are not predicted by the linear analysis). The velocity field is initialized through the following functions:

$$
\begin{array}{ll}
u_{x}=U_{0} \tanh \left[\alpha\left(0.25-\left|\frac{y}{L}-0.5\right|\right)\right], & \forall y \\
u_{y}=U_{0} \delta \sin \left[2 \pi\left(\frac{x}{L}+0.25\right)\right], \quad \forall x
\end{array}
$$

where $U_{0}$ is the mean flow speed, $\alpha$ controls the thickness of the shear layer and $\delta$ determines the amplitude of the perturbation.

In the present work, Grad's approximation is used to reduce spurious oscillations generated by the initialization step $\frac{15[108 \text { : }}{\text { : }}$

$$
f_{\alpha}(t=0) \approx f_{\alpha}^{(e q), 4}+f_{\alpha}^{(1)}
$$

where the non-equilibrium part $f_{\alpha}^{(1)}$ is computed as

$$
f_{\alpha}^{(1)}=\frac{1}{2 c_{s}^{4}} \boldsymbol{a}_{2}^{(1)}: \mathcal{H}_{2, \alpha},
$$

using

$$
\boldsymbol{a}_{2}^{(1)}=-\rho c_{s}^{2} \tau\left(\boldsymbol{u} \otimes \nabla+\boldsymbol{u} \otimes \nabla^{T}\right)
$$

the latter being evaluated through a second-order, centered finite-difference approximation. Looking at the 


\begin{tabular}{||c|c|}
\hline$\nu \delta_{t} / \delta_{x}^{2}$ & $\eta_{\max } \delta_{t} / \delta_{x}^{2}$ \\
\hline \hline $1 \times 10^{-5}$ & $1 \times 10^{-3}$ \\
$5 \times 10^{-5}$ & $1 \times 10^{-3}$ \\
$1 \times 10^{-4}$ & $1 \times 10^{-3}$ \\
$5 \times 10^{-4}$ & $5 \times 10^{-3}$ \\
$1 \times 10^{-3}$ & $1 \times 10^{-2}$ \\
\hline
\end{tabular}

TABLE I: Values of $\eta_{\max } \delta_{t} / \delta_{x}^{2}$ used for the LKS-1 configuration

Chapman-Enskog equations, this expression corresponds to a first-order approximation of the population, $f_{\alpha} \approx$ $f_{\alpha}^{(0)}+\epsilon f_{\alpha}^{(1)}$, with a leading-order error of $\epsilon^{2} f_{\alpha}^{(2)}$. This fact is important as it leads to non-negligible dissipation at the start of the simulation for low-resolution cases 109 .

To establish a non-linear stability map similar to the one derived through the VN stability analysis, simulations for different sets of parameters (Mach number, nondimensional viscosity, and second relaxation coefficient) have been systematically conducted at three different resolutions, namely $128 \times 128,256 \times 256$ and $384 \times 384$. The studies are performed using three different resolutions. As pointed out in a previous study 50 , the fully periodic boundary conditions result in a simulation spanning a limited area of the Fourier space (i.e., $k_{x}$ and $k_{y}$ between $\pi$ and $2 \pi / N$, where $N \geq 2$ is the number of grid-points), effectively filtering out some of the higher wavenumber instabilities. The maximum velocity in $\mathrm{LB}$ units is changed in each case to match the corresponding Mach number, the amplitude of the perturbation is kept constant at 0.05 (changes in $\delta$ lead to significantly higher maximum Mach numbers in the domain), and $\alpha$ takes on values between 80 and 600 , resulting in an initial shear layer thickness as thin as the grid-size. Consequently, the presence of long wavenumber and under-resolved features is forced in these simulations, in order to increase the probability of getting spurious vortices $\frac{105 / 106}{\text {. }}$

For LKS only two scenarios for the second viscosity (free parameter) are considered, namely $\eta \delta_{t} / \delta_{x}^{2}=\eta_{\max } \delta_{t} / \delta_{x}^{2}$ and $0.05, \eta_{\max } \delta_{t} / \delta_{x}^{2}$ being the value corresponding to the widest stability area. The values used for $\eta_{\max } \delta_{t} / \delta_{x}^{2}$ are given in Table I. The resulting stability domains are depicted in Fig. 99, The stability of each configuration was assessed by monitoring the kinetic energy over $50 t_{c}$. As shown in this figure, the SRT collision operator is not stable for non-dimensional viscosities below $5 \times 10^{-5}$. For the LKS operator on the other hand, simulations were stable down to $1 \times 10^{-5}$. Looking at the large gap between the von Neumann stability domain and that obtained via the double periodic shear layer test case, and putting the results next to previous sections on dispersion-dissipation behavior, it can be observed that non-linear effects have a more pronounced effect on the stability area at lower viscosities for the LKS configuration with the free parameter tuned for optimal linear stability. For the other LKS model (with the second viscosity fixed at 0.05), the DPSL stability domain

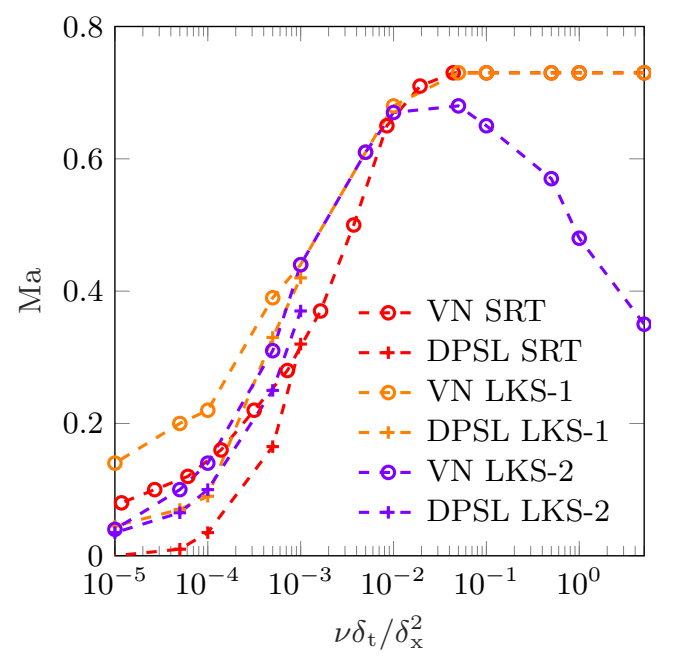

FIG. 9: von Neumann stability domain (VN) vs. stability domain for the double periodic shear layer (DPSL) test-case for the SRT operator and the LKS with maximum linear stability (LKS-1) and $\eta \delta_{t} / \delta_{x}^{2}=0.05($ LKS-2).

is relatively close to its corresponding linear stability domain, as the larger value of the free parameter damps the kinetic modes much faster; as seen in Table $\square$ the free parameters in LKS-1 are always smaller than 0.05.

\section{B. 3-D Taylor-Green vortex}

The 3-D Taylor-Green vortex configuration is another fundamental canonical problem to study vortex dynamics and turbulent flow transition. This problem consists of an all-around periodic cubic simulation domain, initialized using the following equations:

$$
\begin{array}{rlrl}
u_{x} & = & U_{0} \sin \left(x^{*}\right) \cos \left(y^{*}\right) \cos \left(z^{*}\right), & (35 \mathrm{a}) \\
u_{y} & = & -U_{0} \cos \left(x^{*}\right) \sin \left(y^{*}\right) \cos \left(z^{*}\right), & (35 \mathrm{~b}) \\
u_{z} & = & 0, \\
p & \left.=p_{0}+\frac{\rho_{0} U_{0}^{2}}{16}\left[\cos \left(2 z^{*}\right)+2\right]\left[\cos \left(2 x^{*}\right)+\cos \left(2 y^{*}\right)\right] 35 \mathrm{~d}\right)
\end{array}
$$

where normalized coordinates are computed as $\left(x^{*}, y^{*}, z^{*}\right)=(x / L, y / L, z / L)$. As for the previous test-case, populations are initialized using Grad's approximation.

To better observe and clarify the effects of the free parameter on both the stability domain and dissipation of kinetic modes, this configuration, with a Reynolds number $\operatorname{Re}=1600$ and fixing $U_{0}$ at 0.1 , is modeled at three different resolutions, i.e. $32^{3}, 64^{3}$ and $128^{3}$, for different values of the second relaxation coefficient. The results are then compared to well-resolved simulations (i.e. $384^{3}$ and $512^{3}$ ) using the SRT operator. In all cases the simulations are conducted over a period of $30 t_{c}$, where the characteristic time is defined as $t_{c}=L / U_{0}$. The energy spectra 
were computed using the ANAFLAME library ${ }^{110}$ where the energy spectrum tensor $E_{i j}$ is computed through:

$$
E_{i j}(\boldsymbol{k})=\frac{1}{(2 \pi)^{3}} \iiint e^{-j \boldsymbol{k} \cdot \boldsymbol{x}} R_{i j}(\boldsymbol{x}) d \boldsymbol{x}
$$

where the correlation tensor $R_{i j}(\boldsymbol{x})$ in homogeneous turbulence is defined as:

$$
R_{i j}(\boldsymbol{x})=\overline{u_{i}^{\prime}\left(\boldsymbol{x}_{\mathbf{0}}\right) u_{j}^{\prime}\left(\boldsymbol{x}_{\mathbf{0}}+\boldsymbol{x}\right)},
$$

where $\boldsymbol{u}^{\prime}$ is the velocity fluctuation, $\boldsymbol{x}_{\mathbf{0}}$ position in space and $\boldsymbol{x}$ the distance and the overbar is an average over space.

Looking at the energy spectra at $t=10 t_{c}$ displayed in Fig. 10, it is readily observed that the choice of the free parameter, $\lambda$, in agreement with theoretical analyses presented in previous sections, only affects higher wavenumbers. The effects of higher-order moments dissipation for under-resolved simulation are clearly observable in Fig. 11, where the iso-surfaces of the $z$-component of the vorticity for the 15 different configurations studied in this section are displayed (only the upper left quadrant is shown). Indeed, for smaller values of the free parameter, the flow field is polluted by large wavenumber features, most probably caused by dispersion error of small-scale under-resolved features. This can be clearly seen by comparing these iso-surfaces with the one obtained from the $512^{3}$ resolution simulation, displayed in Fig 12. Given that for all three considered resolutions the grid-size is larger than the smallest scale, large wavenumber features (under-resolved with $k \geq \pi / 2$ ) are created and - if not dissipated - will pollute numerically the flow field. The presence of under-resolved features in these simulations is further demonstrated by looking at the energy spectra of the reference simulations (resolutions $384^{3}$ and $\left.512^{3}\right)$.

\section{CONCLUSIONS AND DISCUSSION}

In this work, following a previous study on the asymptotic limit of the lattice kinetic scheme where the effects of introducing the additional term in the EDF on higher order dynamics were established, the behavior of the scheme for non-vanishing wavenumbers is studied through a von Neumann analysis of the linearized collision operator. Through a systematic study of the imaginary part of the eigenvalues (i.e., growth rate), the linear stability domain is established. Furthermore, by comparing the results from different orders of the EDF and different choices of the free parameter in the LKS collision operator, configurations resulting in optimal linear stability properties are presented. It is shown that the use of a fourth-order EDF along with the appropriate choice of the free parameter can have a non-negligible effect on the stability domain of the solver, especially for vanishing non-dimensional viscosities. To further quantify the impact of the EDF on the linear stability of LBMs, it is planned to extend the present study to exact $12 \mid 102$ and approximated $103 / 104$ EDFs derived from the principle of maximum entropy. Corresponding results will be presented in a future paper.

Dispersion and dissipation curves of the LKS collision operator for different values of the free parameter are also studied to clarify its performance for simulations with large non-dimensional physical viscosities. These curves show that an appropriate choice of the free parameter can improve such simulation in two ways: (a) increasing dissipation at higher wavenumbers (where the scheme exhibits inconsistent dispersion properties resulting in the generation of spurious waves), and (b) improving dispersion properties. Guidelines for the choice of the free parameter in these cases are given. In the limit of vanishing non-dimensional physical viscosities, the linear analysis shows that dispersion curves are not affected by the choice of the free parameter. In the dissipation curves, however, larger dissipation of the kinetic modes is observed for larger values of the free parameter. Above a certain threshold (around $3 \pi / 4$ ) the convective mode becomes unstable.

Now that the effects of the free parameter on linear stability, dispersion and dissipation of different modes (acoustic, convective, and kinetic) for non-vanishing wavenumbers have been established, the free parameter can be tied to a local flow-state indicator to guarantee stability and optimal dispersion-dissipation. As an example, the entropic stabilizer following the $\mathrm{KBC}^{13 / 14}$ formulation could be a potential candidate to automatically determine locally the optimal value of the free parameter.

\section{ACKNOWLEDGMENTS}

The financial support of the German research foundation (DFG), Germany within the graduate college for "Micro-Macro-Interactions in Structured Media and Particle Systems" (GRK 1554) is gratefully acknowledged. Furthermore S.A.H. would like to thank G. Janiga for providing the ANAFLAME library source code.
${ }^{1}$ F. Schornbaum and U. Rüde, SIAM J. Sci. Comput 38, C96 (2016)

${ }^{2}$ T. Krüger, H. Kusumaatmaja, A. Kuzmin, O. Shardt, G. Silva, and E. M. Viggen, The Lattice Boltzmann
Method: Principles and Practice (Springer International Publishing, 2017).

3 S. Succi, The Lattice Boltzmann Equation: For Complex States of Flowing Matter (Oxford University Press, 2018). 

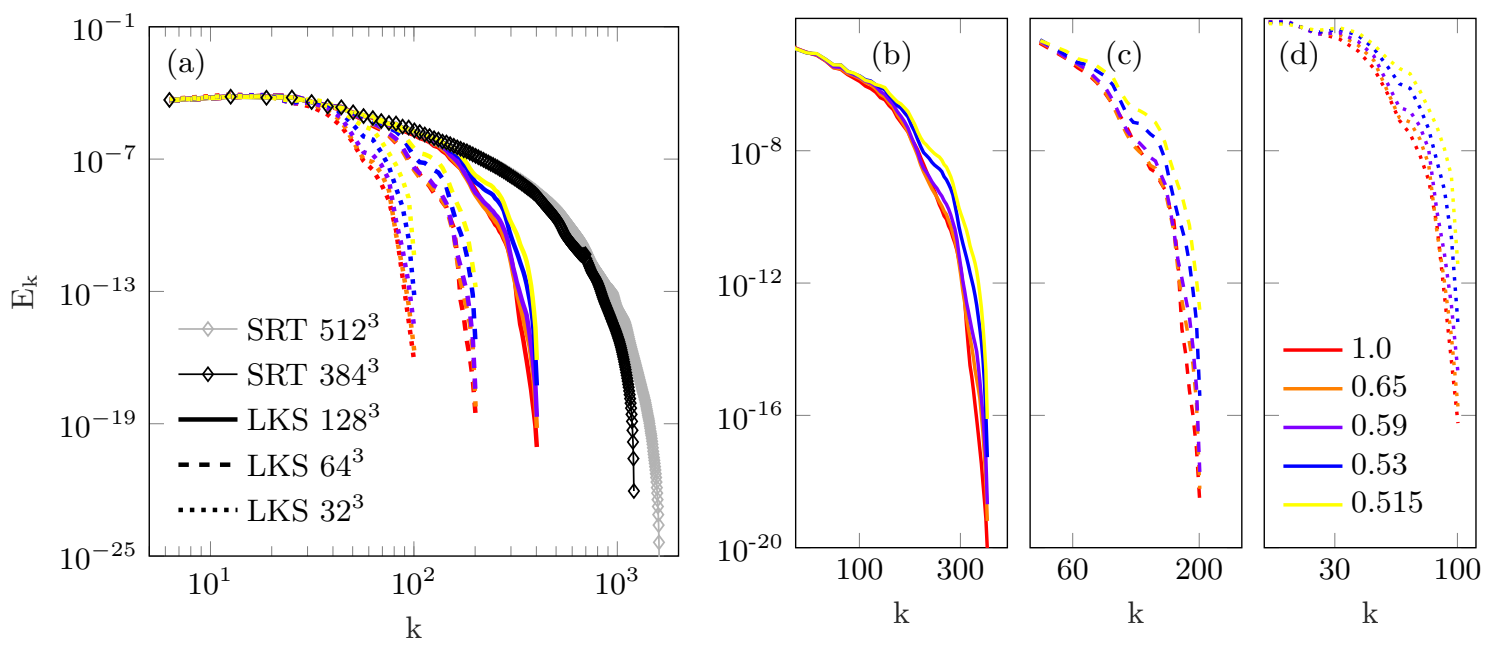

FIG. 10: Energy spectrum for Taylor-Green vortex at $t_{c}=10$ for different spatial resolutions, and values of the free parameter $\lambda$.

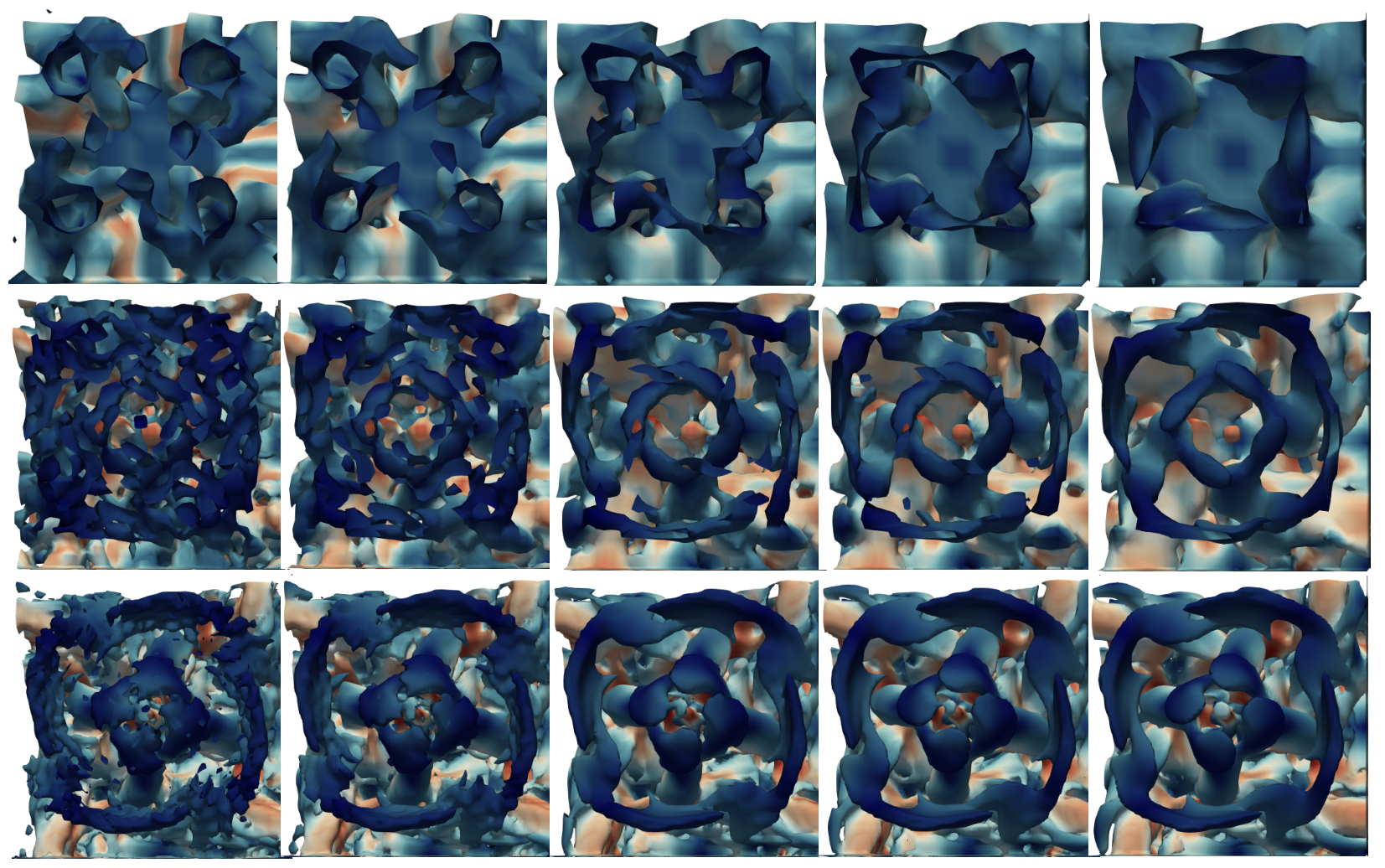

FIG. 11: Iso-surfaces of the $z$-component of vorticity $\omega_{z}=0$ (bottom view, only the upper left quadrant is shown) at $t=10 t_{c}$ obtained using the LKS at three different resolutions (from top to bottom): $32^{3}, 64^{3}, 128^{3}$ with five different values for the free parameter (from left to right): $\lambda=0.515,0.53,0.59,0.65,1$.

${ }^{4}$ C. Lin, K. H. Luo, L. Fei, and S. Succi, Scientific reports 7, 14580 (2017).

5 H. Safari, M. H. Rahimian, and M. Krafczyk, Physical Review E 88, 013304 (2013).

${ }^{6}$ H. Safari, M. Krafczyk, and M. Geier, Computers \& Fluids (2018).

7 S. Hosseini, A. Eshghinejadfard, N. Darabiha, and D. Thévenin, Comput. Math. Appl.

(2017), 10.1016/j.camwa.2017.08.045

S. A. Hosseini, N. Darabiha, and D. Thévenin, International Journal of Heat and Mass Transfer 132, 906 (2019).

9 C. Coreixas, B. Chopard, and J. Latt, arXiv preprint arXiv:1904.12948 (2019).

${ }^{10}$ I. V. Karlin, A. Ferrante, and H. C. Öttinger, Europhys. 


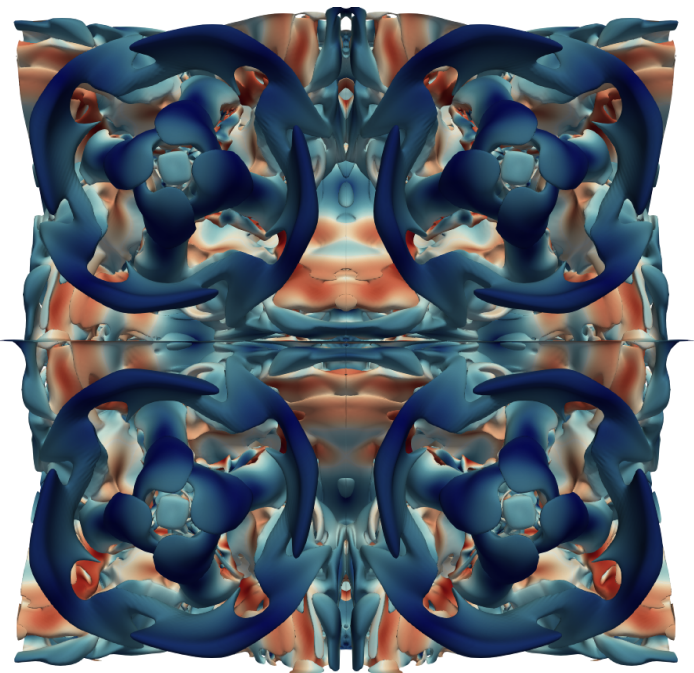

FIG. 12: Iso-surfaces of the $z$-component of vorticity $\omega_{z}=0$ (bottom view) at $t=10 t_{c}$ obtained using the SRT at resolutions $512^{3}$

Lett. 47, $182(1999)$

i1 B. M. Boghosian, J. Yepez, P. V. Coveney, and A. Wager, Proc. Royal Soc. A 457, 717 (2001).

12 S. Ansumali, I. V. Karlin, and H. C. Öttinger, Europhys. Lett. 63, 798 (2003)

${ }^{13}$ I. V. Karlin, F. Bösch, and S. S. Chikatamarla, Phys. Rev. E 90, 031302 (2014)

${ }^{14}$ F. Bösch, S. S. Chikatamarla, and I. V. Karlin, Phys. Rev. E 92, 043309 (2015)

${ }^{15}$ K. K. Mattila, L. A. Hegele, and P. C. Philippi, Phys. Rev. E 91, 063010 (2015)

16 B. Dorschner, N. Frapolli, S. S. Chikatamarla, and I. V. Karlin, Phys. Rev. E 94, 053311 (2016).

17 M. Atif, P. K. Kolluru, C. Thantanapally, and S. Ansumali, Phys. Rev. Lett. 119, 240602 (2017)

18 B. Dorschner, S. S. Chikatamarla, and I. V. Karlin, Phys. Rev. E 97, 023305 (2018)

19 D. d'Humières, Prog. Astronaut. Aeronaut. 159, 450 (1992).

${ }^{20}$ P. Lallemand and L.-S. Luo, Phys. Rev. E 61, 6546 (2000)

21 P. J. Dellar, J. Stat. Mech. Theor. Exp. 06, P06003 (2009).

${ }^{22}$ J. Wang, D. Wang, P. Lallemand, and L.-S. Luo, Comput. Math. Appl. 65, 262 (2013).

${ }^{23}$ J. Miranda Fuentes, F. Kuznik, K. Johannes, and J. Virgone, Phys. Lett. A 378, 374 (2014)

24 Q. Li, Q. Kang, M. Francois, Y. He, and K. Luo, Int. J. Heat Mass Transfer 85, 787 (2015)

${ }^{25}$ K. Suga, Y. Kuwata, K. Takashima, and R. Chikasue, Comput. Math. Appl. 69, 518 (2015).

26 A. Fakhari, D. Bolster, and L.-S. Luo, J. Comput. Phys. 341, 22 (2017)

${ }^{27}$ M. Grasinger, S. Overacker, and J. Brigham, Comput. Fluids 166, 253 (2018)

${ }^{28}$ I. Ginzburg, F. Verhaeghe, and D. d'Humières, Commun. Comput. Phys. 3, 427 (2008)

${ }^{29}$ I. Ginzburg, F. Verhaeghe, and D. d'Humières, Commun. Comput. Phys. 3, 519 (2008)

so A. Kuzmin, I. Ginzburg, and A. Mohamad, Comput.
Math. Appl. 61, 3417 (2011).

${ }^{31}$ G. Silva and V. Semiao, Phys. Rev. E 96, 013311 (2017)

${ }^{32}$ M. Geier, A. Greiner, and J. G. Korvink, Phys. Rev. E 73, 066705 (2006).

${ }^{33}$ M. Geier, A. Greiner, and J. G. Korvink, Eur. Phys. J. Spec. Top. 171, 55 (2009).

${ }^{34}$ D. Lycett-Brown and K. H. Luo, Comput. Math. Appl. 67, 350 (2014).

35 F. Dubois, T. Février, and B. Graille, Commun. Comput. Phys. 17, 10881112 (2015).

${ }^{36}$ F. Dubois, T. Février, and B. Graille, C. R. Mécanique 343, 599 (2015)

${ }^{3}$ L. Fei and K. H. Luo, Int. J. Therm. Sci. 132, 368 (2018).

38 A. De Rosis and K. H. Luo, Phys. Rev. E 99, 013301 (2019).

$3 y$ A. De Rosis, R. Huang, and C. Coreixas, submitted to Phys. Rev. E (2019).

40 J. Latt and B. Chopard, Math. Comput. Simul. 72, 165 (2006).

${ }^{41}$ H. Chen, R. Zhang, I. Staroselsky, and M. Jhon, Physica A 362, 125 (2006)

${ }^{42}$ R. Zhang, X. Shan, and H. Chen, Phys. Rev. E 74, 046703 (2006)

${ }^{43}$ X.-D. Niu, S.-A. Hyodo, T. Munekata, and K. Suga, Phys. Rev. E 76, 036711 (2007)

44 G. Silva and V. Semiao, J. Fluid Mech. 698, 282 (2012)

${ }^{45}$ L. Wang, B. Shi, and Z. Chai, Phys. Rev. E 92, 043311 (2015).

40 Y. Ba, N. Wang, H. Liu, Q. Li, and G. He, Phys. Rev. E 97, 033307 (2018).

4 O. Malaspinas, ArXiv e-prints (2015)

48 F. Brogi, O. Malaspinas, B. Chopard, and C. Bonadonna, J. Acoust. Soc. Am 142, 2332 (2017).

49 C. Coreixas, G. Wissocq, G. Puigt, J.-F. Boussuge, and P. Sagaut, Phys. Rev. E 96, 033306 (2017)

50 C. Coreixas, High-order extension of the recursive regularized lattice Boltzmann method, Ph.D. thesis, INP Toulouse (2018).

51 M. Geier, M. Schönherr, A. Pasquali, and M. Krafczyk, Comput. Math. Appl. 70, 507 (2015)

52 M. Geier, A. Pasquali, and M. Schönherr, J. Comput. Phys. 348, 862 (2017)

53 M. Geier, A. Pasquali, and M. Schönherr, J. Comput. Phys. 348, 889 (2017)

${ }^{54}$ M. Geier and A. Pasquali, Comput. Fluids 166, 139 (2018).

${ }^{55}$ M. Watari and M. Tsutahara, Phys. Rev. E 67, 036306 (2003)

${ }_{50}$ T. Kataoka and M. Tsutahara, Phys. Rev. E 69, 035701 (2004).

57 T. Kataoka and M. Tsutahara, Phys. Rev. E 69, 056702 (2004).

50 M. Watari, Physica A 382, 502 (2007)

59 R. Surmas, C. E. Pico Ortiz, and P. C. Philippi, Eur. Phys. J. Special Topics 171, 81 (2009)

${ }^{\text {ou }}$ S. Ubertini, G. Bella, and S. Succi, Phys. Rev. E 68, 016701 (2003).

${ }^{61}$ M. Sbragaglia and K. Sugiyama, Phys. Rev. E 82, 046709 (2010).

${ }^{62}$ Z. Guo, K. Xu, and R. Wang, Phys. Rev. E 88, 033305 (2013).

os S. Xiao, Commun. Numer. Methods Eng. 23, 71 (2007).

64 Y. Gan, A. Xu, G. Zhang, X. Yu, and Y. Li, Physica A: Statistical Mechanics and its Applications 387, 1721 
(2008).

65 C. Feng, X. Ai-Guo, Z. Guang-Cai, and L. Ying-Jun, Communications in Theoretical Physics 54, 1121 (2010).

66 Y. Bo, P. Wang, Z. Guo, and L.-P. Wang, Computers \& Fluids 155, 9 (2017).

67 T. Inamuro, Philos. Trans. R . Soc. London, Ser. A 360, 477 (2002).

68 L. Wang, J. Mi, X. Meng, and Z. Guo, Commun. Comput. Phys. 17, 908 (2015).

69 M. Yoshino, Y.-H. Hotta, T. Hirozane, and M. Endo, J. Non-Newton. Fluid. Mech. 147, 69 (2007).

70 C.-H. Wang and J.-R. Ho, Physica A 387, 4740 (2008).

71 Y. Kataoka and T. Inamuro, Philos. Trans. R . Soc. London, Ser. A 369, 2528 (2011).

72 T. Inamuro, T. Yokoyama, K. Tanaka, and M. Taniguchi, Comput. Fluids 137, 55 (2016).

73 T. Inamuro and T. Ogata, Phil. Trans. R. Soc. Lond. A 362, 1735 (2004).

74 T. Inamuro, T. Echizen, and F. Horai, Comput. Fluids 175, 83 (2018).

75 L. Wang, J. Mi, and Z. Guo, Int. J. Heat Mass Transfer 94, 269 (2016).

76 X. Meng and Z. Guo, Phys. Rev. E 92, 043305 (2015).

77 X. Meng and Z. Guo, Int. J. Heat Mass Transfer 100, 767 (2016).

78 S. A. Hosseini, N. Darabiha, and D. Thévenin, Phys. Rev. E 99, 023305 (2019).

79 J. Perko and R. A. Patel, Phys. Rev. E 89, 053309 (2014).

80 S. A. Hosseini, N. Darabiha, and D. Thévenin, Physica A 499, 40 (2018).

81 L. Wang, W. Zhao, and X.-D. Wang, Phys. Rev. E 98, 033308 (2018).

${ }^{82}$ X. Yang, B. Shi, and Z. Chai, Phys. Rev. E 90, 013309 (2014).

83 W. Zhao, L. Wang, and W.-A. Yong, Physica A 492, 1570 (2018).

84 S. A. Hosseini, N. Darabiha, D. Thévenin, and A. Eshghinejadfard, Int. J. Mod. Phys. C 28, 1750141 (2017).

85 J. G. Charney, R. Fjörtoft, and J. v. Neumann, Tellus 2, 237 (1950).

86 J. D. Sterling and S. Chen, J. Comput. Phys. 123, 196 (1996).

R. A. Worthing, J. Mozer, and G. Seeley, Phys. Rev. E 56, 2243 (1997).

88 S. Marié, D. Ricot, and P. Sagaut, J. Comput. Phys. 228, 1056 (2009)

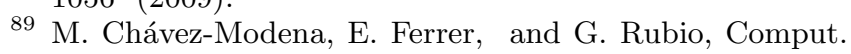

Fluids 172, 397 (2018)

90 G. Wissocq, P. Sagaut, and J.-F. Boussuge, J. Comput. Phys. 380, 311 (2019)

${ }^{91}$ H. Grad, Commun. Pure Appl. Math. 2, 331 (1949)

92 X. Shan and X. He, Phys. Rev. Lett. 80, 65 (1998).

93 X. Shan, X.-F. Yuan, and H. Chen, J. Fluid Mech. 550, $413(2006)$

${ }^{94}$ P. Lallemand and L.-S. Luo, Phys. Rev. E 68, 036706 (2003).

y5 D. N. Siebert, L. A. Hegele, and P. C. Philippi, Phys. Rev. E 77, 026707 (2008).

${ }^{90}$ D. Ricot, S. Mari, P. Sagaut, and C. Bailly, J. Comput. Phys. 228, 4478 (2009)

9r I. Ginzburg, D. d'Humières, and A. Kuzmin, J. Stat. Phys. 139, 1090 (2010)

${ }^{98}$ H. Xu, O. Malaspinas, and P. Sagaut, J. Comput. Phys. 231, 7335 (2012)

99 S. A. Hosseini, N. Darabiha, D. Thévenin, and A. Eshghinejadfard, Int. J. Mod. Phys. C 28, 1750141 (2017)

100 G. V. Krivovichev and S. A. Mikheev, Int. J. Comput. Methods 16, 1850087 (2018).

${ }^{101}$ C. Coreixas et al., under preparation (2019).

102 P. Asinari and I. V. Karlin, Phys. Rev. E 79, 036703 (2009).

${ }^{103}$ N. Frapolli, S. S. Chikatamarla, and I. V. Karlin, Phys. Rev. E 92, 061301 (2015).

${ }^{104}$ M. Atif, M. Namburi, and S. Ansumali, Phys. Rev. E 98, 053311 (2018).

105 D. L. Brown and M. L. Minion, Performance of Underresolved Two-Dimensional Incompressible Flow Simulations, Tech. Rep. (Technical Report Los Alamos National Laboratory, 1995).

106 M. L. Minion and D. L. Brown, J. Comput. Phys. 138, 734 (1997).

107 D. Ricot, S. Marié, P. Sagaut, and C. Bailly, J. Comput. Phys. 228, 4478 (2009).

108 P. A. Skordos, Phys. Rev. E 48, 4823 (1993).

109 K. K. Mattila, L. A. Hegele Jr, and P. C. Philippi, Phys. Rev. E 91, 063010 (2015).

110 C. Zistl, R. Hilbert, G. Janiga, and D. Thévenin, Comput. Vis. Sci. 12, 383 (2009).

111 X. Shan and H. Chen, Int. J. Mod. Phys. C 18, 635 (2007)

112 P. J. Dellar, Phys. Rev. E 65, 036309 (2002).

113 G. Házi and P. Kávrán, J. Phys. A Math. Gen. 39, 3127 (2006)

${ }^{114}$ P. J. Dellar, J. Comput. Phys. 259, 270 (2014)

\section{Appendix A: Relationship with other collision operators}

Owing to the fact that the LKS equilibrium state was designed to recover the correct viscous stress tensor, while the relaxation coefficient $\lambda$ was used to freely control the relaxation of high-order moments, one may wonder if the LKS model could not be related to an already existing collision model that would accomplish the same task. To find this link, let us start from the LKS collision model (8):

$$
\begin{aligned}
\Omega_{\alpha}^{\mathrm{LKS}} & =-\frac{1}{\lambda}\left(f_{\alpha}-f_{\alpha}^{(e q), \mathrm{LKS}}\right) \\
& =-\frac{1}{\lambda}\left(f_{\alpha}-f_{\alpha}^{(e q)}\right)+\frac{A}{\lambda(A-\lambda)} \frac{1}{2 c_{s}^{4}}\left(\boldsymbol{a}_{2}-\boldsymbol{a}_{2}^{(e q)}\right): \mathcal{H}_{2, \alpha},
\end{aligned}
$$

where the second RHS term only impacts the relaxation of the nonequilibium second-order moment of populations, i.e, the viscous stress tensor. All third- and fourth-order moments are then relaxed through the free parameter $\lambda$. 
This kind of behavior is quite common in the LB community, and is usually expressed in the form of a MRT collision model. By rewritting Eq. A1, one obtains

$$
\Omega_{\alpha}^{\mathrm{LKS}}=-\sum_{n=0}^{N} \frac{1}{\tau_{n}} \frac{1}{n ! c_{s}^{2 n}}\left(\boldsymbol{a}_{n}-\boldsymbol{a}_{n}^{(e q), \mathrm{LKS}}\right): \boldsymbol{H}_{n, \alpha},
$$

where $\tau_{n}$ is the relaxation coefficient related to Hermite coefficients of order $n$. More specifically, $\tau_{2}=\tau$ and $\tau_{3}=\tau_{4}=\lambda$ for the LKS model (values of $\tau_{0}$ and $\tau_{1}$ are only relevant in the context of external forcing 28 ). From Eq. A2, one clearly recognizes the MRT collision model expressed in the Hermite polynomial expansion framework that was originally introduced by Shan and Chen 111 . This relationship is very important since it means that all systematic derivations, that were obtained in the context of Hermite polynomial expansion, can be applied to the LKS approach. In other words, the extension to any kind of physics or lattice can be done in a straightforward manner ${ }^{93}$.

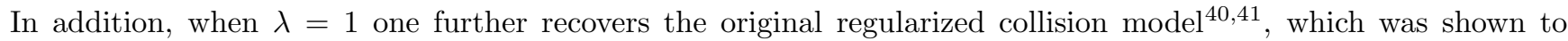
improve the stability of LBMs in various configurations 43.46. This might explain why a value of $\lambda$ close to 1 helped improving the numerical stability of the LKS model without impacting its accuracy ${ }^{78}$.

\section{Appendix B: Jacobian of Hermite coefficients at equilibrium}

Hereafter, Jacobian expressions of high-order Hermite coefficients at equilibrium $\boldsymbol{a}_{n}^{(e q)}(n>2)$ are derived. Starting with third-order coefficients at equilibrium,

$$
\partial_{f_{\beta}} \boldsymbol{a}_{3}^{(e q)}=-2 \frac{\boldsymbol{a}_{1}^{(e q)} \otimes \boldsymbol{a}_{1}^{(e q)} \otimes \boldsymbol{a}_{1}^{(e q)}}{\left(\boldsymbol{a}_{0}^{(e q)}\right)^{3}}+\frac{\boldsymbol{c}_{\beta} \otimes \boldsymbol{a}_{1}^{(e q)} \otimes \boldsymbol{a}_{1}^{(e q)}+\boldsymbol{a}_{1}^{(e q)} \otimes \boldsymbol{c}_{\beta} \otimes \boldsymbol{a}_{1}^{(e q)}+\boldsymbol{a}_{1}^{(e q)} \otimes \boldsymbol{a}_{1}^{(e q)} \otimes \boldsymbol{c}_{\beta}}{\left(\boldsymbol{a}_{0}^{(e q)}\right)^{2}} .
$$

Following the same steps, the Jacobian of the fourth-order coefficients can be expressed as

$$
\begin{aligned}
& \partial_{f_{\beta}} \boldsymbol{a}_{4}^{(e q)}=-3 \frac{\boldsymbol{a}_{1}^{(e q)} \otimes \boldsymbol{a}_{1}^{(e q)} \otimes \boldsymbol{a}_{1}^{(e q)} \otimes \boldsymbol{a}_{1}^{(e q)}}{\left(\boldsymbol{a}_{0}^{(e q)}\right)^{3}} \\
& +\frac{\boldsymbol{c}_{\beta} \otimes \boldsymbol{a}_{1}^{(e q)} \otimes \boldsymbol{a}_{1}^{(e q)} \otimes \boldsymbol{a}_{1}^{(e q)}+\boldsymbol{a}_{1}^{(e q)} \otimes \boldsymbol{c}_{\beta} \otimes \boldsymbol{a}_{1}^{(e q)} \otimes \boldsymbol{a}_{1}^{(e q)}+\boldsymbol{a}_{1}^{(e q)} \otimes \boldsymbol{a}_{1}^{(e q)} \otimes \boldsymbol{c}_{\beta} \otimes \boldsymbol{a}_{1}^{(e q)}+\boldsymbol{a}_{1}^{(e q)} \otimes \boldsymbol{a}_{1}^{(e q)} \otimes \boldsymbol{a}_{1}^{(e q)} \otimes \boldsymbol{c}_{\beta}}{\left(\boldsymbol{a}_{0}^{(e q)}\right)^{2}} .
\end{aligned}
$$

\section{Appendix C: Effect of higher-order Hermite polynomials in the EDF on dispersion-dissipation}

The off-diagonal component of the stress tensor recovered by the classical lattice Boltzmann formulation with the SRT collision operator and a second-order EDF are known to deviate from the Newtonian fluid stress tensor for large values of the Mach number following $\mathcal{O}\left(\mathrm{Ma}^{3}\right)$. An asymptotic analysis shows that introducing the third-order Hermite polynomial in the EDF can eliminate the leading-order error term in the stress tensor $138 / 47 / 49112$ 114. This effect can also be observed in the spectral behavior of the scheme, namely the spectral speed and dissipation $\frac{50101}{10}$. This fact is illustrated in Fig. 13 for three different Mach numbers, i.e. Ma=0.1, 0.3 and 0.5 for a non-dimensional viscosity of $\nu \delta_{t} / \delta_{x}^{2}=1 \times 10^{-2}$. 

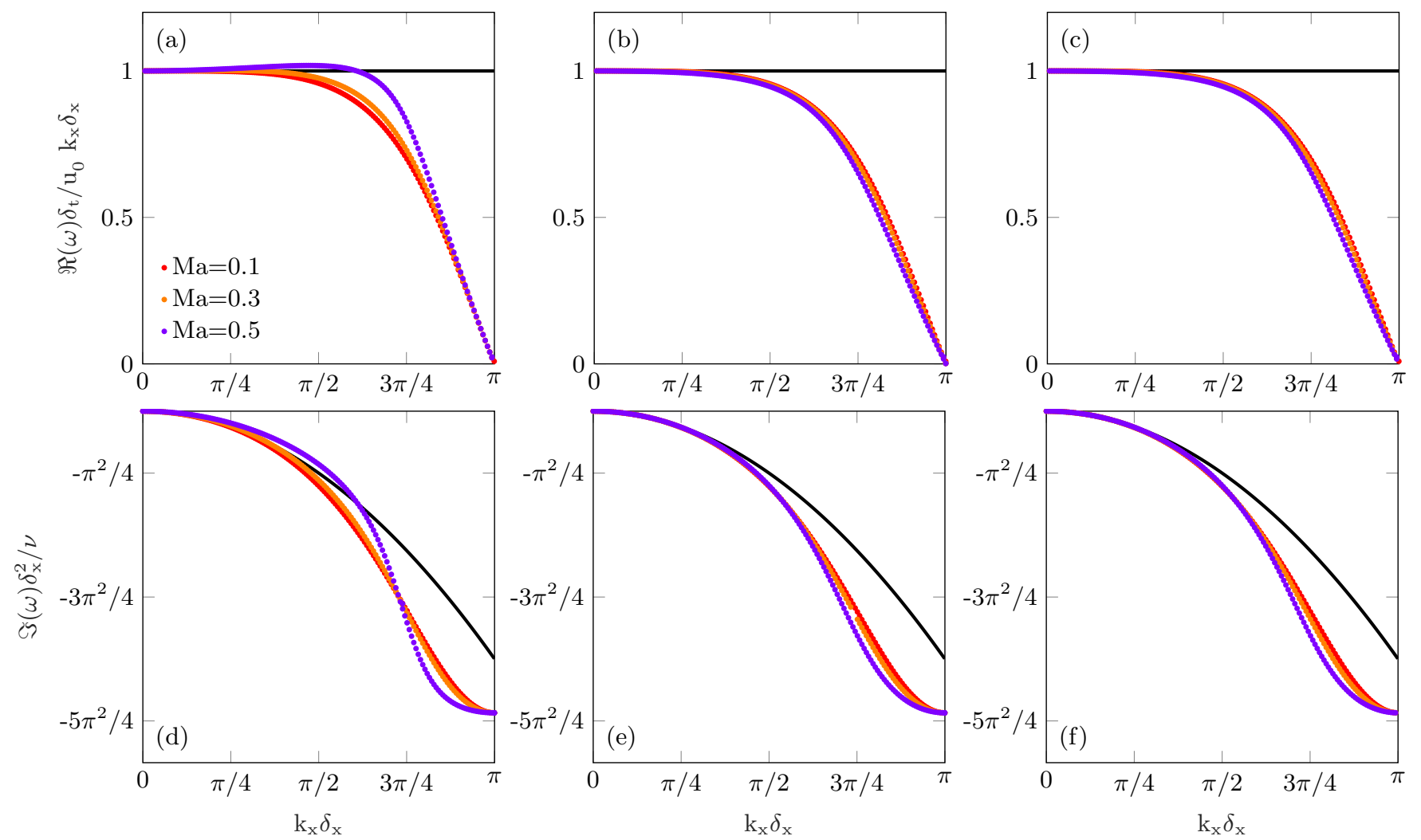

FIG. 13: Effect of the Mach number on propagation speed ( $a, b$ and $c$ ) and dissipation ( $d$, e and $f$ ) for SRT collision operator with (from left to right): second, third and fourth-order EDFs 